\title{
Some quantum vertex algebras of Zamolodchikov-Faddeev type
}

\author{
Martin Karel and Haisheng Lil \\ Department of Mathematical Sciences, Rutgers University, Camden, NJ 08102
}

\begin{abstract}
This is a continuation of a previous study of quantum vertex algebras of Zamolodchikov-Faddeev type. In this paper, we focus our attention on the special case associated to diagonal unitary rational quantum Yang-Baxter operators. We prove that the associated weak quantum vertex algebras, if not zero, are irreducible quantum vertex algebras with a normal basis in a certain sense.
\end{abstract}

\section{Introduction}

In an attempt to associate vertex algebra-like structures with quantum affine algebras, in [Li6] we studied general formal vertex operators (=quantum fields) on an arbitrary vector space, to see what kind of algebraic structures they could possibly "generate". More specifically, we studied what we called "quasi compatible" sets of formal vertex operators on a vector space and we proved that any quasi compatible set generates a nonlocal vertex algebra in a certain canonical way. (Whereas vertex algebras are analogues of commutative and associative algebras, nonlocal vertex algebras are analogues of noncommutative associative algebras.) It follows from this general result that to most of the interesting algebras one can associate nonlocal vertex algebras. For example, for any highest weight module $W$ for a quantum affine algebra, the generating functions in Drinfeld's realization form a quasi compatible set of vertex operators on $W$ and therefore they generate a nonlocal vertex algebra.

As general nonlocal vertex algebras are too "wild", one hopes to get "better" families of nonlocal vertex algebras from "better" families of vertex operators. Motivated by the $\mathcal{S}$-locality axiom in Etingof-Kazhdan's notion of quantum vertex operator algebra [EK], we then continued to study what we called $\mathcal{S}$-local sets and quasi $\mathcal{S}$-local sets of vertex operators. We proved that from a quasi $\mathcal{S}$-local set of vertex operators, indeed one can get a better nonlocal vertex algebra whose adjoint vertex operators form an $\mathcal{S}$-local set of vertex operators (on the nonlocal vertex algebra). Motivated by this result and by Etingof-Kazhdan's notion of quantum vertex operator algebra we then formulated and studied a notion of weak quantum vertex algebra and a notion of quantum vertex algebra.

A weak quantum vertex algebra (over $\mathbb{C}$ ) is a vector space $V$ equipped with a distinguished vector 1 and a linear map $Y: V \rightarrow \operatorname{Hom}(V, V((x)))$ satisfying the condition that $Y(\mathbf{1}, x)=1$ (the identity operator on $V$ ),

$$
Y(v, x) \mathbf{1} \in V[[x]] \text { and }\left.(Y(v, x) \mathbf{1})\right|_{x=0}=v \quad \text { for } v \in V,
$$

\footnotetext{
${ }^{1}$ Partially supported by NSA grant H98230-05-1-0018 and NSF grant DMS-0600189
} 
and that for any $u, v \in V$, there exist $u^{(i)}, v^{(i)} \in V, f_{i}(x) \in \mathbb{C}((x)), i=1, \ldots, r$, such that

$$
\begin{aligned}
& x_{0}^{-1} \delta\left(\frac{x_{1}-x_{2}}{x_{0}}\right) Y\left(u, x_{1}\right) Y\left(v, x_{2}\right) \\
& \quad-x_{0}^{-1} \delta\left(\frac{x_{2}-x_{1}}{-x_{0}}\right) \sum_{i=1}^{r} f_{i}\left(-x_{0}\right) Y\left(v^{(i)}, x_{2}\right) Y\left(u^{(i)}, x_{1}\right) \\
= & x_{2}^{-1} \delta\left(\frac{x_{1}-x_{0}}{x_{2}}\right) Y\left(Y\left(u, x_{0}\right) v, x_{2}\right) .
\end{aligned}
$$

A quantum vertex algebra is a weak quantum vertex algebra $V$ equipped with a unitary rational quantum Yang-Baxter operator (with one parameter)

$$
\mathcal{S}(x): V \otimes V \rightarrow V \otimes V \otimes \mathbb{C}((x))
$$

such that for any $u, v \in V$, (1.1) holds with

$$
\mathcal{S}(x)(v \otimes u)=\sum_{i=1}^{r} v^{(i)} \otimes u^{(i)} \otimes f_{i}(x)
$$

and such that some other conditions hold. The notion of quantum vertex algebra generalizes the notions of vertex algebra and vertex superalgebra.

The designation of the notion of weak quantum vertex algebra was motivated by the following conceptual result. Let $W$ be any vector space and set $\mathcal{E}(W)=$ $\operatorname{Hom}(W, W((x)))$. A subset $T$ of $\mathcal{E}(W)$ is said to be $\mathcal{S}$-local if for any $a(x), b(x) \in T$, there exist (finitely many)

$$
a^{(i)}(x), b^{(i)}(x) \in T, f_{i}(x) \in \mathbb{C}((x)), i=1, \ldots, r
$$

such that

$$
\left(x_{1}-x_{2}\right)^{k} a\left(x_{1}\right) b\left(x_{2}\right)=\left(x_{1}-x_{2}\right)^{k} \sum_{i=1}^{r} f_{i}\left(x_{2}-x_{1}\right) b^{(i)}\left(x_{2}\right) a^{(i)}\left(x_{1}\right)
$$

for some nonnegative integer $k$. Let $T$ be an $\mathcal{S}$-local subset of $\mathcal{E}(W)$. For $a(x), b(x) \in$ $T$ with the above information, we define

$$
\begin{aligned}
& Y_{\mathcal{E}}\left(a(x), x_{0}\right) b(x)=\operatorname{Res}_{x_{1}} x_{0}^{-1} \delta\left(\frac{x_{1}-x}{x_{0}}\right) a\left(x_{1}\right) b(x) \\
& -\operatorname{Res}_{x_{1}} x_{0}^{-1} \delta\left(\frac{x-x_{1}}{-x_{0}}\right) \sum_{i=1}^{r} f_{i}\left(x_{2}-x_{1}\right) b^{(i)}\left(x_{2}\right) a^{(i)}\left(x_{1}\right) .
\end{aligned}
$$

It was proved in [Li6] that any $\mathcal{S}$-local subset of $\mathcal{E}(W)$ generates a weak quantum vertex algebra with $W$ as a canonical module. This particular result generalizes a 
result of [Li1], which states that for any vector space $W$, every local subset of $\mathcal{E}(W)$ generates a vertex algebra with $W$ as a module (see [Li2], [Li3], [Li7], and [Li10] for similar results).

Regarding quantum vertex algebras, a variant, which was formulated in [Li6], of ([EK], Proposition 1.11), is that if a weak quantum vertex algebra $V$ is nondegenerate in the sense of [EK], $V$ is a quantum vertex algebra with $\mathcal{S}(x)$ uniquely determined. Furthermore, it was proved in [Li8] (cf. [Li4] for vertex algebras) that if a nonlocal vertex algebra $V$ (over $\mathbb{C}$ ) is of countable dimension and if $V$ as a (left) $V$-module is irreducible, then $V$ is nondegenerate.

In [Li8], to demonstrate how to use the general construction theorem which was established in [Li6], we constructed some quantum vertex algebras by using algebras of Zamolodchikov-Faddeev type (see [ZZ], [F]). More specifically, let $H$ be a finite-dimensional vector space equipped with a bilinear form $\langle\cdot, \cdot\rangle$ and let $\mathcal{S}(x): H \otimes H \rightarrow H \otimes H \otimes \mathbb{C}[[x]]$ be a linear map. We studied weak quantum vertex algebras $V$ equipped with a linear map $\phi: H \rightarrow V$ such that $V$ is generated by $\phi(H)$, satisfying the relations for $u, v \in H$ :

$$
\begin{aligned}
& Y\left(\phi(u), x_{1}\right) Y\left(\phi(v), x_{2}\right)-\sum_{i=1}^{r} f_{i}\left(x_{2}-x_{1}\right) Y\left(\phi\left(v^{(i)}\right), x_{2}\right) Y\left(\phi\left(u^{(i)}\right), x_{1}\right) \\
= & \langle u, v\rangle x_{2}^{-1} \delta\left(\frac{x_{1}}{x_{2}}\right)
\end{aligned}
$$

where $\mathcal{S}(x)(v \otimes u)=\sum_{i=1}^{r} v^{(i)} \otimes u^{(i)} \otimes f_{i}(x)$. We then constructed a universal weak quantum vertex algebra $V(H, \mathcal{S})$ and proved that $V(H, \mathcal{S})$ is nondegenerate for some special cases, so that $V(H, \mathcal{S})$ is a quantum vertex algebra (see Section 4). (If $\phi$ is injective and if $V(H, \mathcal{S})$ is nondegenerate, then $\mathcal{S}(x)$ is necessarily a unitary rational quantum Yang-Baxter operator.)

This current paper is a natural continuation of our studies on weak quantum vertex algebras $V(H, \mathcal{S})$. In this paper, we focus our attention on the special case in which $\mathcal{S}(x)$ is "diagonal". Let $\mathbf{Q}(x)=\left(q_{i j}(x)\right)$ be an $l \times l$ matrix in $\mathbb{C}[[x]]$ with $q_{i j}(x) q_{j i}(-x)=1$ for $1 \leq i, j \leq l$. Take $H$ to be a $2 l$-dimensional vector space with a basis $\left\{u^{(i)}, v^{(i)} \mid 1 \leq i \leq l\right\}$, equip $H$ with the bilinear form $\langle\cdot, \cdot\rangle$ defined by

$$
\left\langle u^{(i)}, u^{(j)}\right\rangle=0=\left\langle v^{(i)}, v^{(j)}\right\rangle, \quad\left\langle u^{(i)}, v^{(j)}\right\rangle=\delta_{i j}=-q_{i i}(0)\left\langle v^{(j)}, u^{(i)}\right\rangle
$$

for $1 \leq i, j \leq l$, and define $\mathcal{S}(x): H \otimes H \rightarrow H \otimes H \otimes \mathbb{C}[[x]]$ by

$$
\begin{aligned}
& \mathcal{S}(x)\left(u^{(j)} \otimes u^{(i)}\right)=u^{(j)} \otimes u^{(i)} \otimes q_{i j}(x), \quad \mathcal{S}(x)\left(v^{(j)} \otimes v^{(i)}\right)=v^{(j)} \otimes v^{(i)} \otimes q_{i j}(x), \\
& \mathcal{S}(x)\left(v^{(j)} \otimes u^{(i)}\right)=v^{(j)} \otimes u^{(i)} \otimes q_{j i}(-x), \\
& \mathcal{S}(x)\left(u^{(j)} \otimes v^{(i)}\right)=u^{(j)} \otimes v^{(i)} \otimes q_{j i}(-x)
\end{aligned}
$$

for $1 \leq i, j \leq l$. For this pair $(H, \mathcal{S})$, the associated universal weak quantum vertex algebra $V(H, \mathcal{S})$ is alternatively denoted by $V_{\mathbf{Q}(x)}$. Our main goal is to determine the structure and establish the nondegeneracy of $V_{\mathbf{Q}(x)}$. As one of the main results in this paper, we prove that $V_{\mathbf{Q}(x)}$ is either zero or irreducible as a $V_{\mathbf{Q}(x)}$-module 
with a normal basis in a certain sense, so that it is a nondegenerate quantum vertex algebra.

As a technical step, we first study the case with $\mathbf{Q}(x)=\mathbf{Q}=\left(q_{i j}\right)$ a complex matrix with $q_{i j} q_{j i}=1$ for $1 \leq i, j \leq l$. We define an associative algebra $\mathcal{A}_{\mathbf{Q}}$ with generators $X_{i, m}, Y_{i, m}$ for $1 \leq i \leq l, m \in \mathbb{Z}$, subject to relations

$$
\begin{gathered}
X_{i, m} X_{j, n}=q_{i j} X_{j, n} X_{i, m}, \quad Y_{i, m} Y_{j, n}=q_{i j} Y_{j, n} Y_{i, m} \\
X_{i, m} Y_{j, n}-q_{j i} Y_{j, n} X_{i, m}=\delta_{i j} \delta_{m+n+1,0}
\end{gathered}
$$

for $1 \leq i, j \leq l, m, n \in \mathbb{Z}$. If $q_{i j}=1$ for all $i, j=1, \ldots, l, \mathcal{A}_{\mathbf{Q}}$ is a Weyl algebra and if $q_{i j}=-1$ for all $i, j=1, \ldots, l, \mathcal{A}_{\mathbf{Q}}$ is a Clifford algebra. (In general, we have $q_{i i}= \pm 1$ for $1 \leq i \leq l$, so that $\mathcal{A}_{\mathbf{Q}}$ contains Weyl algebras or Clifford algebras as subalgebras.) Let $V_{\mathbf{Q}}$ denote the quotient module of the regular left $\mathcal{A}_{\mathbf{Q}}$-module modulo the left ideal generated by $X_{i, m}, Y_{i, m}$ for $1 \leq i \leq l, m \geq 0$. It is proved that $V_{\mathbf{Q}}$ is irreducible and there is a canonical simple quantum vertex algebra structure on $V_{\mathbf{Q}}$. Furthermore, $V_{\mathbf{Q}}$ has a conformal vector of central charge $l$. Note that quantum coordinate algebras, closely related to $\mathcal{A}_{\mathbf{Q}}$, have appeared before in the study of noncommutative geometry (cf. [Ma1, [Ma2]) and physics noncommutative field theory (cf. $[\mathrm{Ku}]$ ). Our emphasis in this study is on quantum vertex algebras and modules.

To achieve our results for the nonconstant case with $\mathbf{Q}(x)=\left(q_{i j}(x)\right)$, we make use of a certain filtration. We define an increasing filtration $F=\left\{F_{n}\right\}_{n \geq 0}$ of $V_{\mathbf{Q}(x)}$ by using the canonical generators and we prove that if the weak quantum vertex algebra $V_{\mathbf{Q}(x)}$ is nonzero, the associated graded weak quantum vertex algebra $\operatorname{Gr}_{F}\left(V_{\mathbf{Q}(x)}\right)$ is isomorphic to the irreducible quantum vertex algebra $V_{\mathbf{Q}(0)}$. Then we prove that $V_{\mathbf{Q}(x)}$, if not zero, is an irreducible quantum vertex algebra. Furthermore, we show that for certain cases, $V_{\mathbf{Q}(x)}$ is indeed nonzero.

One of the authors (H. Li) would like to thank Igor Frenkel for a discussion on quantum vertex algebras and Zamolodchikov-Faddeev algebras. Some time ago, he and J. Ding had some similar ideas. Part of this paper was done during H.Li's visit in January 2007 at the Chern Institute of Mathematics, Nankai University, Tianjin, China. We would like to thank Professors Chengming Bai and Weiping Zhang for their hospitality.

This paper is organized as follows: In Section 2, we present some basic results about general nonlocal vertex algebras. In Section 3, we study the associative algebras $\mathcal{A}_{\mathbf{Q}}$ and quantum vertex algebras $V_{\mathbf{Q}}$. In Section 4 , we study quantum vertex algebras $V_{\mathbf{Q}(x)}$.

\section{Some results for general nonlocal vertex alge- bras}

In this section we recall the notions of nonlocal vertex algebra, weak quantum vertex algebra, and quantum vertex algebra, and we present some basic results on increasing 
filtrations for general nonlocal vertex algebras associated with a generating subset.

We start by recalling the definition of a nonlocal vertex algebra from [Li6] (cf. $[\mathrm{Ka},[\mathrm{BK}],[\mathrm{Li} 3]$ ). A nonlocal vertex algebra is a vector space $V$, equipped with a linear map

$$
\begin{aligned}
Y: V & \rightarrow \operatorname{Hom}(V, V((x))) \subset(\operatorname{End} V)\left[\left[x, x^{-1}\right]\right] \\
v & \mapsto Y(v, x)=\sum_{n \in \mathbb{Z}} v_{n} x^{-n-1} \quad\left(v_{n} \in \operatorname{End} V\right)
\end{aligned}
$$

and equipped with a distinguished vector $\mathbf{1}$, satisfying the condition that for $v \in V$

$$
\begin{aligned}
& Y(\mathbf{1}, x) v=v \\
& Y(v, x) \mathbf{1} \in V[[x]] \quad \text { and } \quad \lim _{x \rightarrow 0} Y(v, x) \mathbf{1}=v
\end{aligned}
$$

and the condition that for $u, v, w \in V$, there exists a nonnegative integer $l$ such that

$$
\left(x_{0}+x_{2}\right)^{l} Y\left(u, x_{0}+x_{2}\right) Y\left(v, x_{2}\right) w=\left(x_{0}+x_{2}\right)^{l} Y\left(Y\left(u, x_{0}\right) v, x_{2}\right) w .
$$

Following [EK], let $Y(x): V \otimes V \rightarrow V((x))$ be the linear map associated with $Y$.

For a nonlocal vertex algebra $V$, let $\mathcal{D}$ be the linear operator on $V$ defined by $\mathcal{D}(v)=v_{-2} \mathbf{1}$ for $v \in V$. Then

$$
[\mathcal{D}, Y(v, x)]=Y(\mathcal{D} v, x)=\frac{d}{d x} Y(v, x) \quad \text { for } v \in V
$$

Furthermore, we have $Y(v, x) \mathbf{1}=e^{x \mathcal{D}} v$ and $\mathcal{D}(\mathbf{1})=0$ for $v \in V$.

For a nonlocal vertex algebra $V$, a $V$-module is a vector space $W$ equipped with a linear map $Y_{W}: V \rightarrow \operatorname{Hom}(W, W((x)))$ satisfying the condition that

$$
Y_{W}(\mathbf{1}, x)=1_{W} \quad(\text { the identity operator on } W)
$$

and for $u, v \in V, w \in W$, there exists $l \in \mathbb{N}$ such that

$$
\left(x_{0}+x_{2}\right)^{l} Y_{W}\left(u, x_{0}+x_{2}\right) Y_{W}\left(v, x_{2}\right) w=\left(x_{0}+x_{2}\right)^{l} Y_{W}\left(Y\left(u, x_{0}\right) v, x_{2}\right) w .
$$

Next, we recall from [Li6] the notions of weak quantum vertex algebra and quantum vertex algebra. A weak quantum vertex algebra is a vector space $V$ (over $\mathbb{C}$ ) equipped with a distinguished vector 1 and a linear map $Y$ from $V$ to $\operatorname{Hom}(V, V((x))))$, satisfying the condition that

$$
\begin{aligned}
& Y(\mathbf{1}, x)=1 \\
& Y(v, x) \mathbf{1} \in V[[x]] \quad \text { and } \quad \lim _{x \rightarrow 0} Y(v, x) \mathbf{1}=v
\end{aligned}
$$


for $v \in V$, and that for any $u, v \in V$, there exist $u^{(i)}, v^{(i)} \in V, f_{i}(x) \in \mathbb{C}((x)), i=$ $1, \ldots, r$, such that

$$
\begin{aligned}
& x_{0}^{-1} \delta\left(\frac{x_{1}-x_{2}}{x_{0}}\right) Y\left(u, x_{1}\right) Y\left(v, x_{2}\right) \\
& \quad-x_{0}^{-1} \delta\left(\frac{x_{2}-x_{1}}{-x_{0}}\right) \sum_{i=1}^{r} f_{i}\left(-x_{0}\right) Y\left(v^{(i)}, x_{2}\right) Y\left(u^{(i)}, x_{1}\right) \\
= & x_{2}^{-1} \delta\left(\frac{x_{1}-x_{0}}{x_{2}}\right) Y\left(Y\left(u, x_{0}\right) v, x_{2}\right) .
\end{aligned}
$$

Alternatively, a weak quantum vertex algebra is a nonlocal vertex algebra that satisfies $\mathcal{S}$-locality (cf. [EK]) in the sense that for any $u, v \in V$, there exist $u^{(i)}, v^{(i)} \in$ $V, f_{i}(x) \in \mathbb{C}((x)), i=1, \ldots, r$, such that

$$
\left(x_{1}-x_{2}\right)^{k} Y\left(u, x_{1}\right) Y\left(v, x_{2}\right)=\left(x_{1}-x_{2}\right)^{k} \sum_{i=1}^{r} f_{i}\left(x_{2}-x_{1}\right) Y\left(v^{(i)}, x_{2}\right) Y\left(u^{(i)}, x_{1}\right)
$$

for some nonnegative integer $k$ (depending on $u$ and $v$ ).

The notion of quantum vertex algebra involves a quantum Yang-Baxter operator. A unitary rational quantum Yang-Baxter operator with one parameter on a vector space $U$ is a linear map

$$
\mathcal{S}(x): U \otimes U \rightarrow U \otimes U \otimes \mathbb{C}((x))
$$

such that

$$
\begin{aligned}
\mathcal{S}(x) \mathcal{S}^{21}(-x) & =1 \\
\mathcal{S}^{12}\left(x_{1}\right) \mathcal{S}^{13}\left(x_{1}-x_{2}\right) \mathcal{S}^{23}\left(x_{2}\right) & =\mathcal{S}^{23}\left(x_{2}\right) \mathcal{S}^{13}\left(x_{1}-x_{2}\right) \mathcal{S}^{12}\left(x_{1}\right)
\end{aligned}
$$

A quantum vertex algebra (cf. [EK]) is a weak quantum vertex algebra $V$ equipped with a unitary rational quantum Yang-Baxter operator

$$
\mathcal{S}(x): V \otimes V \rightarrow V \otimes V \otimes \mathbb{C}((x))
$$

such that for $u, v \in V,(2.8)$ holds with $\mathcal{S}(x)(v \otimes u)=\sum_{i=1}^{r} v^{(i)} \otimes u^{(i)} \otimes f_{i}(x)$ and such that

$$
\begin{aligned}
& {[\mathcal{D} \otimes 1, \mathcal{S}(x)]=-\frac{d}{d x} \mathcal{S}(x)} \\
& \mathcal{S}(z)(Y(x) \otimes 1)=(Y(x) \otimes 1) \mathcal{S}^{23}(z) \mathcal{S}^{13}(z+x)
\end{aligned}
$$

Remark 2.1. A nonlocal vertex algebra $V$ is said to be nondegenerate (see [EK]) if for every positive integer $n$, the linear map

$$
Z_{n}: \mathbb{C}\left(\left(x_{1}\right)\right) \cdots\left(\left(x_{n}\right)\right) \otimes V^{\otimes n} \rightarrow V\left(\left(x_{1}\right)\right) \cdots\left(\left(x_{n}\right)\right)
$$


defined by

$$
Z_{n}\left(f \otimes v^{(1)} \otimes \cdots \otimes v^{(n)}\right)=f Y\left(v^{(1)}, x_{1}\right) \cdots Y\left(v^{(n)}, x_{n}\right) \mathbf{1}
$$

is injective. It was proved in [Li8] that if $V$ is of countable dimension (over $\mathbb{C}$ ) and if $V$ as a (left) $V$-module is irreducible, then $V$ is nondegenerate.

Remark 2.2. A variation of a theorem of Etingof-Kazhdan ([EK], Proposition 1.11), formulated in [Li6], is that if a weak quantum vertex algebra $V$ is nondegenerate, then $\mathcal{S}$-locality defines a unitary rational quantum Yang-Baxter operator $\mathcal{S}(x)$ on $V$ such that $(V, \mathcal{S}(x))$ is a quantum vertex algebra and this $\mathcal{S}(x)$ is the unique quantum Yang-Baxter operator to make $V$ a quantum vertex algebra. In view of this, the term "a nondegenerate quantum vertex algebra," or "an irreducible quantum vertex algebra" (without reference to a quantum Yang-Baxter operator) is unambiguous.

We shall need the following result of [LL] (Proposition 4.5.7, which can be extended for nonlocal vertex algebras with the same proof):

Lemma 2.3. Let $V$ be a nonlocal vertex algebra, let $W$ be a $V$-module, and let $u, v \in V, p, q \in \mathbb{Z}, w \in W$. Then there exist nonnegative integers $l$ and $m$ such that

$$
u_{p} v_{q} w=\sum_{i=0}^{m} \sum_{j=0}^{l}\left(\begin{array}{c}
p-l \\
i
\end{array}\right)\left(\begin{array}{l}
l \\
j
\end{array}\right)\left(u_{p-l-i+j} v\right)_{q+l+i-j} w .
$$

We shall also need the following analogue:

Lemma 2.4. Let $V$ be a nonlocal vertex algebra and let $u, v, w \in V, m, n \in \mathbb{Z}$. There exist nonnegative integers $l$ and $k$ such that

$$
\left(u_{m} v\right)_{n} w=\sum_{i=0}^{k} \sum_{j \geq 0}\left(\begin{array}{c}
-l \\
i
\end{array}\right)\left(\begin{array}{c}
m+i \\
j
\end{array}\right)(-1)^{j} u_{m+l+i-j} v_{n-l-i+j} w .
$$

Proof. From definition, there exists a nonnegative integer $l$ such that

$$
\left(x_{0}+x_{2}\right)^{l} Y\left(Y\left(u, x_{0}\right) v, x_{2}\right) w=\left(x_{0}+x_{2}\right)^{l} Y\left(u, x_{0}+x_{2}\right) Y\left(v, x_{2}\right) w .
$$

We have

$$
\begin{aligned}
\left(u_{m} v\right)_{n} w & =\operatorname{Res}_{x_{0}} \operatorname{Res}_{x_{2}} x_{0}^{m} x_{2}^{n} Y\left(Y\left(u, x_{0}\right) v, x_{2}\right) w \\
& =\operatorname{Res}_{x_{0}} \operatorname{Res}_{x_{2}} x_{0}^{m} x_{2}^{n}\left(x_{2}+x_{0}\right)^{-l}\left(\left(x_{2}+x_{0}\right)^{l} Y\left(Y\left(u, x_{0}\right) v, x_{2}\right) w\right) \\
& =\operatorname{Res}_{x_{0}} \operatorname{Res}_{x_{2}} \sum_{i \geq 0}\left(\begin{array}{c}
-l \\
i
\end{array}\right) x_{0}^{m+i} x_{2}^{n-l-i}\left(\left(x_{2}+x_{0}\right)^{l} Y\left(Y\left(u, x_{0}\right) v, x_{2}\right) w\right) .
\end{aligned}
$$


Let $k$ be a nonnegative integer such that $x_{0}^{m+k} Y\left(u, x_{0}\right) v \in V\left[\left[x_{0}\right]\right]$. Then

$$
\begin{aligned}
& \left(u_{m} v\right)_{n} w \\
= & \operatorname{Res}_{x_{0}} \operatorname{Res}_{x_{2}} \sum_{i=0}^{k}\left(\begin{array}{c}
-l \\
i
\end{array}\right) x_{0}^{m+i} x_{2}^{n-l-i}\left(\left(x_{2}+x_{0}\right)^{l} Y\left(Y\left(u, x_{0}\right) v, x_{2}\right) w\right) \\
= & \operatorname{Res}_{x_{0}} \operatorname{Res}_{x_{2}} \sum_{i=0}^{k}\left(\begin{array}{c}
-l \\
i
\end{array}\right) x_{0}^{m+i} x_{2}^{n-l-i}\left(\left(x_{0}+x_{2}\right)^{l} Y\left(u, x_{0}+x_{2}\right) Y\left(v, x_{2}\right) w\right) \\
= & \operatorname{Res}_{x_{1}} \operatorname{Res}_{x_{2}} \sum_{i=0}^{k}\left(\begin{array}{c}
-l \\
i
\end{array}\right)\left(x_{1}-x_{2}\right)^{m+i} x_{2}^{n-l-i} x_{1}^{l} Y\left(u, x_{1}\right) Y\left(v, x_{2}\right) w \\
= & \operatorname{Res}_{x_{1}} \operatorname{Res}_{x_{2}} \sum_{i=0}^{k}\left(\begin{array}{c}
-l \\
i
\end{array}\right) \sum_{j \geq 0}\left(\begin{array}{c}
m+i \\
j
\end{array}\right)(-1)^{j} x_{1}^{m+l+i-j} x_{2}^{n-l-i+j} Y\left(u, x_{1}\right) Y\left(v, x_{2}\right) w \\
= & \sum_{i=0}^{k}\left(\begin{array}{c}
-l \\
i
\end{array}\right) \sum_{j \geq 0}\left(\begin{array}{c}
m+i \\
j
\end{array}\right)(-1)^{j} u_{m+l+i-j} v_{n-l-i+j} w,
\end{aligned}
$$

as desired.

Let $V$ be a nonlocal vertex algebra and let $T$ be a generating subset of $V$ in the sense that $V$ is the smallest nonlocal vertex subalgebra that contains $T$. Then (see [BK], [Li3]) $V$ is linearly spanned by the vectors

$$
u_{m_{1}}^{(1)} \cdots u_{m_{r}}^{(r)} \mathbf{1}
$$

for $r \geq 0, u^{(1)}, \ldots, u^{(r)} \in T, m_{1}, \ldots, m_{r} \in \mathbb{Z}$.

Remark 2.5. Here, we mention a simple fact which is straightforward to prove. Let $V$ be a nonlocal vertex algebra with a generating subset $T$ and let $K$ be any nonlocal vertex algebra. For any map $f$ from $T$ to $K, f$ extends to at most one nonlocal-vertex-algebra homomorphism from $V$ to $K$. On the other hand, if $\psi$ is a linear map from $V$ to $K$ such that

$$
\psi(Y(a, x) v)=Y(\psi(a), x) \psi(v) \quad \text { for } a \in T, v \in V,
$$

then $\psi$ is a nonlocal-vertex-algebra homomorphism.

In the following we shall associate two increasing filtrations of $V$ to each generating subset.

Lemma 2.6. Let $V$ be a nonlocal vertex algebra and let $T$ be a generating subset. For $n \in \mathbb{N}$, set

$$
E_{n}=\operatorname{span}\left\{u_{m_{1}}^{(1)} \cdots u_{m_{r}}^{(r)} \mathbf{1} \mid 0 \leq r \leq n, u^{(1)}, \ldots, u^{(r)} \in T, m_{1}, \ldots, m_{r} \in \mathbb{Z}\right\} .
$$

Then $E_{n} \subset E_{n+1}$ for $n \in \mathbb{N}, E_{0}=\mathbb{C} \mathbf{1}$, and $\cup_{n \in \mathbb{N}} E_{n}=V$. Furthermore,

$$
a_{k} E_{n} \subset E_{m+n} \quad \text { for } a \in E_{m}, k \in \mathbb{Z}, m, n \in \mathbb{N} .
$$


Proof. From definition, we have $E_{n} \subset E_{n+1}$ for $n \in \mathbb{N}$ and $E_{0}=\mathbb{C} 1$. As $T$ generates $V$, we get $V=\cup_{n \in \mathbb{N}} E_{n}$. With $E_{0}=\mathbb{C} \mathbf{1}$, we see that (2.15) holds for $m=0$. Assume $m \geq 1$. From definition we have

$$
E_{m}=\operatorname{span}\left\{u_{q} E_{m-1} \mid u \in T, q \in \mathbb{Z}\right\}
$$

It follows from induction on $m$ and Lemma 2.4 that (2.15) holds for all $m \in \mathbb{N}$.

Definition 2.7. Let $G$ be a group with identity element e. A nonlocal vertex $G$-graded algebra is a nonlocal vertex algebra $V$ equipped with a $G$-grading $V=$ $\coprod_{g \in G} V[g]$ such that $\mathbf{1} \in V[e]$ and $Y(u, x) v \in V[g h]((x))$ for $u \in V[g], v \in V[h]$ with $g, h \in G$.

Remark 2.8. Let $V$ be a nonlocal vertex algebra and let $E=\left\{E_{n}\right\}_{n \in \mathbb{Z}}$ be an increasing subspace-filtration of $V$ with $1 \in E_{0}$, satisfying the condition that

$$
a_{k} E_{n} \subset E_{m+n} \quad \text { for } a \in E_{m}, m, n, k \in \mathbb{Z}
$$

(cf. $(2.15)$ ). Form the graded vector space

$$
\operatorname{Gr}_{E}(V)=\coprod_{n \in \mathbb{Z}}\left(E_{n} / E_{n-1}\right)
$$

It is straightforward to show (cf. [Li8], Lemma 3.13) that $\operatorname{Gr}_{E}(V)$ is a nonlocal vertex algebra with $\mathbf{1}+E_{-1} \in E_{0} / E_{-1}$ as the vacuum vector and with

$$
\left(a+E_{m-1}\right)_{k}\left(b+E_{n-1}\right)=a_{k} b+E_{m+n-1} \in E_{m+n} / E_{m+n-1}
$$

for $a \in E_{m}, b \in E_{n}, m, n, k \in \mathbb{Z}$. In fact, $\operatorname{Gr}_{E}(V)$ is a nonlocal vertex $\mathbb{Z}$-graded algebra. Furthermore, if $E$ is the filtration associated with a generating subset $T$ of $V$, then $\left\{u+E_{0} \mid u \in T\right\}\left(\subset E_{1} / E_{0}\right)$ is a generating subset of $\operatorname{Gr}_{E}(V)$. A result that was obtained in [Li8] is that if $\operatorname{Gr}_{E}(V)$ is nondegenerate, then $V$ is nondegenerate.

With a generating subset $T$ of $V$ we have another increasing filtration.

Lemma 2.9. Let $V$ be a nonlocal vertex algebra and let $T$ be a generating subset. For $n \in \mathbb{Z}$, let $F_{n}$ be the linear span of the vectors

$$
u_{m_{1}}^{(1)} \cdots u_{m_{r}}^{(r)} \mathbf{1}
$$

for $r \geq 1$ if $n<0$, for $r \geq 0$ if $n \geq 0$, and for $u^{(1)}, \ldots, u^{(r)} \in T, m_{1}, \ldots, m_{r} \in \mathbb{Z}$ with $m_{1}+\cdots+m_{r} \geq-n$. Then $\left\{F_{n}\right\}_{n \in \mathbb{Z}}$ is an increasing filtration of $V$ such that $\mathbf{1} \in F_{0}$ and

$$
a_{m} F_{n} \subset F_{k+n-m-1} \quad \text { for } a \in F_{k}, k, m, n \in \mathbb{Z} \text {. }
$$


Proof. It is clear that $\mathbf{1} \in F_{0}$ and $\left\{F_{n}\right\}_{n \in \mathbb{Z}}$ is an increasing filtration of $V$. For $u \in T, m, n \in \mathbb{Z}$, from definition we have

$$
u_{m} F_{n} \subset F_{n-m}
$$

It follows from induction on $r$ and Lemma 2.4 that for any nonnegative integer $r$, for any $a$ of the form $u_{m_{1}}^{(1)} \cdots u_{m_{r}}^{(r)} \mathbf{1}$ with $u^{(1)}, \ldots, u^{(r)} \in T, m_{1}, \ldots, m_{r} \in \mathbb{Z}$, and for any $m \in \mathbb{Z}$ we have

$$
a_{m} F_{n} \subset F_{n-m-m_{1}-\cdots-m_{r}-1}
$$

Then (2.18) is clear.

A $\mathbb{Z}$-graded nonlocal vertex algebra is a nonlocal vertex algebra $U$ equipped with a $\mathbb{Z}$-grading $U=\coprod_{n \in \mathbb{Z}} U_{(n)}$ such that $\mathbf{1} \in U_{(0)}$ and

$$
u_{k} v \in U_{(m+n-k-1)}
$$

for all $u \in U_{(m)}, v \in U_{(n)}, m, n, k \in \mathbb{Z}$.

Proposition 2.10. Let $F=\left\{F_{n}\right\}_{n \in \mathbb{Z}}$ be an increasing filtration of $V$ with $\mathbf{1} \in F_{0}$ satisfying (2.18). Then the associated graded vector space $\operatorname{Gr}_{F}(V)=\coprod_{n \in \mathbb{Z}}\left(F_{n} / F_{n-1}\right)$ is a $\mathbb{Z}$-graded nonlocal vertex algebra with $\mathbf{1}+F_{-1}$ as the vacuum vector, where

$$
\left(a+F_{m-1}\right)_{k}\left(b+F_{n-1}\right)=a_{k} b+F_{m+n-k-2}
$$

for $a \in F_{m}, b \in F_{n}, m, n, k \in \mathbb{Z}$. Furthermore, if $F$ is the filtration associated with a generating subset $T$ of $V$, then $\left\{u+F_{0} \mid u \in T\right\} \subset F_{1} / F_{0}$ is a generating subset of $\operatorname{Gr}_{F}(V)$.

Proof. It is easy to see that the axioms that involve the vacuum vector, namely the vacuum and creation properties, hold. For weak associativity, we need to show that for $a \in F_{m}, b \in F_{n}, c \in F_{k}$ with $m, n, k \in \mathbb{Z}$, there exists a nonnegative integer $l$ such that

$$
\left(x_{0}+x_{2}\right)^{l} Y\left(\bar{a}, x_{0}+x_{2}\right) Y\left(\bar{b}, x_{2}\right) \bar{c}=\left(x_{0}+x_{2}\right)^{l} Y\left(Y\left(\bar{a}, x_{0}\right) \bar{b}, x_{2}\right) \bar{c}
$$

where $\bar{a}=a+F_{m-1}, \bar{b}=b+F_{n-1}, \bar{c}=c+F_{k-1}$. Let $l$ be a nonnegative integer such that

$$
\left(x_{0}+x_{2}\right)^{l} Y\left(a, x_{0}+x_{2}\right) Y\left(b, x_{2}\right) c=\left(x_{0}+x_{2}\right)^{l} Y\left(Y\left(a, x_{0}\right) b, x_{2}\right) c
$$

which is expanded as

$$
\sum_{p, q \in \mathbb{Z}} \sum_{i \geq 0}\left(\begin{array}{c}
l-p-1 \\
i
\end{array}\right) a_{p} b_{q} c x_{0}^{l-p-1-i} x_{2}^{i-q-1}=\sum_{p^{\prime}, q^{\prime} \in \mathbb{Z}} \sum_{i^{\prime} \geq 0}\left(\begin{array}{l}
l \\
i^{\prime}
\end{array}\right)\left(a_{p^{\prime}} b\right)_{q^{\prime}} c x_{0}^{l-i^{\prime}-p^{\prime}-1} x_{2}^{i^{\prime}-q^{\prime}-1} .
$$


Let $r, s \in \mathbb{Z}$. Extracting the coefficients of $x_{0}^{r} x_{2}^{s}$ from both sides we get

$$
\sum_{i \geq 0}\left(\begin{array}{c}
r+i \\
i
\end{array}\right) a_{l-r-1-i} b_{i-1-s} c=\sum_{i^{\prime} \geq 0}\left(\begin{array}{c}
l \\
i^{\prime}
\end{array}\right)\left(a_{l-i^{\prime}-r-1} b\right)_{i^{\prime}-s-1} c .
$$

With $a \in F_{m}, b \in F_{n}, c \in F_{k}$, by definition we have

$$
\bar{b}_{i-1-s} \bar{c}=b_{i-1-s} c+F_{n+k+s-i-1},
$$

and furthermore,

$$
\bar{a}_{l-r-1-i} \bar{b}_{i-1-s} \bar{c}=a_{l-r-1-i} b_{i-1-s} c+F_{m+n+k+r+s-l-1} .
$$

On the other hand, we have

$$
\bar{a}_{l-i^{\prime}-r-1} \bar{b}=a_{l-i^{\prime}-r-1} b+F_{m+n+r+i^{\prime}-l-1}
$$

and

$$
\left(\bar{a}_{l-i^{\prime}-r-1} \bar{b}\right)_{i^{\prime}-s-1} \bar{c}=\left(a_{l-i^{\prime}-r-1} b\right)_{i^{\prime}-s-1} c+F_{m+n+k+r+s-l-1} .
$$

Then by (2.20) we have

$$
\sum_{i \geq 0}\left(\begin{array}{c}
r+i \\
i
\end{array}\right) \bar{a}_{l-r-1-i} \bar{b}_{i-1-s} \bar{c}=\sum_{i^{\prime} \geq 0}\left(\begin{array}{l}
l \\
i^{\prime}
\end{array}\right)\left(\bar{a}_{l-i^{\prime}-r-1} \bar{b}\right)_{i^{\prime}-s-1} \bar{c}
$$

Multiplying both sides by $x_{0}^{r} x_{2}^{s}$, summing up over all $r, s \in \mathbb{Z}$, and then changing indices we get

$$
\sum_{p, q \in \mathbb{Z}} \sum_{i \geq 0}\left(\begin{array}{c}
l-p-1 \\
i
\end{array}\right) \bar{a}_{p} \bar{b}_{q} \bar{c} x_{0}^{l-p-1-i} x_{2}^{i-q-1}=\sum_{p^{\prime}, q^{\prime} \in \mathbb{Z}} \sum_{i^{\prime} \geq 0}\left(\begin{array}{l}
l \\
i^{\prime}
\end{array}\right)\left(\bar{a}_{p^{\prime}} \bar{b}\right)_{q^{\prime}} \bar{c} x_{0}^{l-i^{\prime}-p^{\prime}-1} x_{2}^{i^{\prime}-q^{\prime}-1}
$$

which gives (2.19). This proves that $\mathrm{Gr}_{F}(V)$ is a nonlocal vertex algebra. It follows from definition that $\operatorname{Gr}_{F}(V)$ is a $\mathbb{Z}$-graded nonlocal vertex algebra.

For the last assertion, from definition we have $T \subset F_{1}$ (as $u=u_{-1} \mathbf{1}$ for $\left.u \in T\right)$. For any $n \in \mathbb{Z}$, consider a typical vector

$$
a=u_{m_{1}}^{(1)} \cdots u_{m_{r}}^{(r)} \mathbf{1} \in F_{n},
$$

where $r \geq 1, u^{(1)}, \ldots, u^{(r)} \in T, m_{1}, \ldots, m_{r} \in \mathbb{Z}$ with $m_{1}+\cdots+m_{r} \geq-n$. If $m_{1}+\cdots+m_{r}>-n$, we have $a \in F_{n-1}$, so that $a+F_{n-1}=0 \operatorname{in}_{G_{F}}(V)$. Assume that $m_{1}+\cdots+m_{r}=-n$. Set $\overline{\mathbf{1}}=\mathbf{1}+F_{-1}$. Note that $u_{m} F_{k} \subset F_{k-m}$ for $u \in T, m, k \in \mathbb{Z}$. From definition we have

$$
a+F_{n-1}=u_{m_{1}}^{(1)} \cdots u_{m_{r}}^{(r)} \mathbf{1}+F_{-m_{1}-\cdots-m_{r}-1}=\bar{u}_{m_{1}}^{(1)} \cdots \bar{u}_{m_{r}}^{(r)} \overline{\mathbf{1}}
$$

Then it follows that $\operatorname{Gr}_{F}(V)$ is generated by $\left\{u+F_{0} \mid u \in T\right\}$. 
Proposition 2.11. Let $V$ be a nonlocal vertex algebra. Assume that there exists a lower-truncated increasing filtration $F=\left\{F_{n}\right\}_{n \in \mathbb{Z}}$ of $V$ with $\mathbf{1} \in F_{0}$, satisfying either (2.15) or (2.18), such that the left adjoint module for $\operatorname{Gr}_{F}(V)$ is graded-irreducible. Then the left adjoint module for $V$ is irreducible.

Proof. Let $U$ be a nonzero $V$-submodule of $V$. We have an increasing filtration $F_{U}=\left\{F_{n} \cap U\right\}_{n \in \mathbb{Z}}$ of $U$. For each $n \in \mathbb{Z}$, the natural map from $F_{n} \cap U$ to $F_{n} / F_{n-1}$ gives rise to an embedding of $\left(F_{n} \cap U\right) /\left(F_{n-1} \cap U\right)$ into $F_{n} / F_{n-1}$. Then we can view $\operatorname{Gr}_{F_{U}}(U)$ as a subspace of $\operatorname{Gr}_{F}(V)$. As $U$ is a $V$-submodule, $\operatorname{Gr}_{F_{U}}(U)$ is a graded $\operatorname{Gr}_{F}(V)$-submodule. Since $\operatorname{Gr}_{F}(V)$ is graded-irreducible, we have either $\operatorname{Gr}_{F_{U}}(U)=$ 0 or $\operatorname{Gr}_{F_{U}}(U)=\operatorname{Gr}_{F}(V)$.

Assume $\operatorname{Gr}_{F_{U}}(U)=0$. Then $F_{n} \cap U=F_{n-1} \cap U$ for all $n \in \mathbb{Z}$. For any $k \in \mathbb{Z}$, we have $F_{k} \cap U=\cup_{n \geq k}\left(F_{n} \cap U\right)=U$, which implies $U \subset F_{k}$. Thus $U \subset \cap_{k \in \mathbb{Z}} F_{k}$. As $F$ is lower-truncated, we have $\cap_{n \in \mathbb{Z}} F_{n}=0$. This contradicts that $U \neq 0$. Therefore, we must have $\operatorname{Gr}_{F_{U}}(U)=\operatorname{Gr}_{F}(V)$. Then $\left(F_{n+1} \cap U\right) /\left(F_{n} \cap U\right)=F_{n+1} / F_{n}$ for $n \in \mathbb{Z}$. That is, $F_{n+1}=\left(F_{n+1} \cap U\right)+F_{n} \subset U+F_{n}$ for all $n \in \mathbb{Z}$. From this we get

$$
F_{n+i} \subset U+F_{n} \quad \text { for } i \geq 0 .
$$

For every $n \in \mathbb{Z}$, as $\cup_{i \geq 0} F_{n+i}=V$, we have $V=U+F_{n}$. Then $V=U$ because $F_{n}=0$ for some $n \in \mathbb{Z}$. This proves that $V$ is irreducible.

Remark 2.12. Let $V=\coprod_{n \in \mathbb{Z}} V_{(n)}$ be a lower truncated $\mathbb{Z}$-graded nonlocal vertex algebra. For $n \in \mathbb{Z}$, set $F_{n}=\coprod_{m<n} V_{(m)}$. Then $\left\{F_{n}\right\}$ is an increasing filtration satisfying (2.18). Furthermore, $\operatorname{Gr}_{F}(V) \simeq V$ as a nonlocal vertex algebra. It follows from Proposition 2.11 that if $V$ is graded irreducible, then $V$ is irreducible.

The following is straightforward:

Lemma 2.13. Let $V$ be a nonlocal vertex $G$-graded algebra with $G$ an abelian group and let $\varepsilon: G \times G \rightarrow \mathbb{C}^{\times}$be a normalized $\mathbb{C}^{\times}$-valued 2 -cocycle of $G$ in the sense that

$$
\begin{aligned}
& \varepsilon(\alpha, 0)=\varepsilon(0, \alpha)=1 \\
& \varepsilon(\alpha, \beta+\gamma) \varepsilon(\beta, \gamma)=\varepsilon(\alpha, \beta) \varepsilon(\alpha+\beta, \gamma) \quad \text { for } \alpha, \beta, \gamma \in G .
\end{aligned}
$$

Define a linear map $Y_{\varepsilon}: V \rightarrow(\operatorname{End} V)\left[\left[x, x^{-1}\right]\right]$ by

$$
Y_{\varepsilon}(u, x) v=\varepsilon(\alpha, \beta) Y(u, x) v \quad \text { for } u \in V[\alpha], v \in V[\beta], \alpha, \beta \in G .
$$

Then $\left(V, Y_{\varepsilon}, \mathbf{1}\right)$ carries the structure of a nonlocal vertex $G$-graded algebra. Furthermore, $\left(V, Y_{\varepsilon}, \mathbf{1}\right)$ is nondegenerate if and only if $V$ is nondegenerate, and $\left(V, Y_{\varepsilon}, \mathbf{1}\right)$ is $G$-graded irreducible if and only if $V$ is G-graded irreducible.

The following is a vertex analogue of a well known construction of associative algebras: 
Proposition 2.14. Let $G$ be a group, let $U$ be a nonlocal vertex algebra on which $G$ acts by automorphisms, and let $V=\coprod_{g \in G} V[g]$ be a nonlocal vertex $G$-graded algebra. Define a linear map

$$
Y_{\sharp}:(U \otimes V) \rightarrow(\operatorname{End}(U \otimes V))\left[\left[x, x^{-1}\right]\right]
$$

by

$$
Y_{\sharp}(u \otimes v, x)\left(u^{\prime} \otimes v^{\prime}\right)=Y(u, x) g\left(u^{\prime}\right) \otimes Y(v, x) v^{\prime}
$$

for $u, u^{\prime} \in U, v \in V[g], g \in G, v^{\prime} \in V$. Then $\left(U \otimes V, Y_{\sharp}, \mathbf{1} \otimes \mathbf{1}\right)$ carries the structure of a nonlocal vertex algebra which we denote by $U \sharp_{G} V$. Furthermore, $U$ and $V$ are subalgebras of $U \sharp_{G} V$ and

$$
Y_{\sharp}\left(v, x_{1}\right) Y_{\sharp}\left(u, x_{2}\right)=Y_{\sharp}\left(g(u), x_{2}\right) Y_{\sharp}\left(v, x_{1}\right)
$$

for $u \in U, v \in V[g]$ with $g \in G$. Moreover, $U \sharp_{G} V$ is a weak quantum vertex algebra if both $U$ and $V$ are weak quantum vertex algebras.

Proof. First, for $u, u^{\prime} \in U, v \in V[g], v^{\prime} \in V$ with $g \in G$ we have

$$
Y_{\sharp}(u \otimes v, x)\left(u^{\prime} \otimes v^{\prime}\right)=Y(u, x) g\left(u^{\prime}\right) \otimes Y(v, x) v^{\prime} \in(U \otimes V)((x)) .
$$

Second, $Y_{\sharp}(\mathbf{1} \otimes \mathbf{1}, x)(u \otimes v)=u \otimes v$ and $Y_{\sharp}(u \otimes v, x)(\mathbf{1} \otimes \mathbf{1})=Y(u, x) \mathbf{1} \otimes Y(v, x) \mathbf{1}$. Let $u, u^{\prime}, u^{\prime \prime} \in U, v \in V[g], v^{\prime} \in V[h], v^{\prime \prime} \in V$ with $g, h \in G$. Then

$$
\begin{aligned}
& Y_{\sharp}\left(u \otimes v, x_{0}+x_{2}\right) Y_{\sharp}\left(u^{\prime} \otimes v^{\prime}, x_{2}\right)\left(u^{\prime \prime} \otimes v^{\prime \prime}\right) \\
= & Y\left(u, x_{0}+x_{2}\right) g Y\left(u^{\prime}, x_{2}\right) h u^{\prime \prime} \otimes Y\left(v, x_{0}+x_{2}\right) Y\left(v^{\prime}, x_{2}\right) v^{\prime \prime} \\
= & Y\left(u, x_{0}+x_{2}\right) Y\left(g u^{\prime}, x_{2}\right) g h u^{\prime \prime} \otimes Y\left(v, x_{0}+x_{2}\right) Y\left(v^{\prime}, x_{2}\right) v^{\prime \prime},
\end{aligned}
$$

and

$$
\begin{aligned}
& Y_{\sharp}\left(Y_{\sharp}\left(u \otimes v, x_{0}\right)\left(u^{\prime} \otimes v^{\prime}\right), x_{2}\right)\left(u^{\prime \prime} \otimes v^{\prime \prime}\right) \\
= & Y_{\sharp}\left(Y\left(u, x_{0}\right) g u^{\prime} \otimes Y\left(v, x_{0}\right) v^{\prime}, x_{2}\right)\left(u^{\prime \prime} \otimes v^{\prime \prime}\right) \\
= & Y\left(Y\left(u, x_{0}\right) g u^{\prime}, x_{2}\right)(g h) u^{\prime \prime} \otimes Y\left(Y\left(v, x_{0}\right) v^{\prime}, x_{2}\right) v^{\prime \prime},
\end{aligned}
$$

as $Y\left(v, x_{0}\right) v^{\prime} \in V[g h]\left(\left(x_{0}\right)\right)$. Then weak associativity follows immediately. This proves that $\left(U \otimes V, Y_{\sharp}, \mathbf{1} \otimes \mathbf{1}\right)$ is a nonlocal vertex algebra. Recall that $\mathbf{1} \in V[e]$ and that $e$ acts on $U$ as identity. It is easy to see that $U$, identified with $U \otimes \mathbf{1}$, and $V$, identified with $\mathbf{1} \otimes V$, are subalgebras of $U \sharp_{G} V$.

For the last assertion, let $u, u^{\prime} \in U, v \in V[g], v^{\prime} \in V$. Then

$$
\begin{aligned}
& Y_{\sharp}\left(\mathbf{1} \otimes v, x_{1}\right) Y_{\sharp}\left(u \otimes \mathbf{1}, x_{2}\right)\left(u^{\prime} \otimes v^{\prime}\right) \\
= & g Y\left(u, x_{2}\right) e u^{\prime} \otimes Y\left(v, x_{1}\right) v^{\prime} \\
= & Y\left(g u, x_{2}\right) g u^{\prime} \otimes Y\left(v, x_{1}\right) v^{\prime} \\
= & Y_{\sharp}\left(g u \otimes \mathbf{1}, x_{2}\right) Y_{\sharp}\left(\mathbf{1} \otimes v, x_{1}\right)\left(u^{\prime} \otimes v^{\prime}\right) .
\end{aligned}
$$


For $u \in U, v \in V$, we have

$$
Y_{\sharp}(u \otimes \mathbf{1}, x)(\mathbf{1} \otimes v)=Y(u, x) \mathbf{1} \otimes v .
$$

It follows that $U \sharp_{G} V$ is generated by $U$ and $V$. Then it is clear that $U \sharp_{G} V$ is a weak quantum vertex algebra if both $U$ and $V$ are weak quantum vertex algebras.

The smash product nonlocal vertex algebra $U \sharp_{G} V$ has the following universal property:

Proposition 2.15. Let $G, U, V$ be given as in Proposition 2.14. Suppose that $K$ is a nonlocal vertex algebra and $\psi: U \rightarrow K$ and $\phi: V \rightarrow K$ are homomorphisms of nonlocal vertex algebras such that

$$
Y\left(\phi(v), x_{1}\right) Y\left(\psi(u), x_{2}\right)=Y\left(\psi(g u), x_{2}\right) Y\left(\phi(v), x_{1}\right)
$$

for $u \in U, v \in V[g]$ with $g \in G$. Then there exists a unique nonlocal vertex algebra homomorphism $f: U \sharp_{G} V \rightarrow K$, extending both $\psi$ and $\phi$.

Proof. As $U \sharp_{G} V$ is generated by $U$ and $V$, the uniqueness follows immediately. For the existence, we define a linear map $\theta: U \sharp_{G} V \rightarrow K$ by

$$
\theta(u \otimes v)=\psi(u)_{-1} \phi(v) \quad \text { for } u \in U, v \in V
$$

It is clear that $\theta$ extends both $\psi$ and $\phi$. It remains to prove that $\theta$ is a homomorphism of nonlocal vertex algebras. For $u \in U, v \in V[g]$, by (2.25) we have

$$
Y\left(\psi(u), x_{2}\right) Y\left(\phi(v), x_{1}\right) \mathbf{1}=Y\left(\phi(v), x_{1}\right) Y\left(\psi\left(g^{-1} u\right), x_{2}\right) \mathbf{1}
$$

which implies that $Y(\psi(u), x) \phi(v) \in K[[x]]$. For any $u \in U, v, v^{\prime} \in V$, there exists a nonnegative integer $l$ such that

$$
\left(x_{0}+x_{2}\right)^{l} Y\left(\psi(u), x_{0}+x_{2}\right) Y\left(\phi(v), x_{2}\right) \phi\left(v^{\prime}\right)=\left(x_{0}+x_{2}\right)^{l} Y\left(Y\left(\psi(u), x_{0}\right) \phi(v), x_{2}\right) \phi\left(v^{\prime}\right) .
$$

As $Y(\psi(u), x) Y\left(\phi(v), x_{2}\right) \phi\left(v^{\prime}\right)$ involves only nonnegative powers of $x$, in fact we have

$$
Y\left(\psi(u), x_{0}+x_{2}\right) Y\left(\phi(v), x_{2}\right) \phi\left(v^{\prime}\right)=Y\left(Y\left(\psi(u), x_{0}\right) \phi(v), x_{2}\right) \phi\left(v^{\prime}\right) .
$$

Furthermore, we have

$$
\begin{aligned}
\theta\left(Y_{\sharp}(u \otimes \mathbf{1}, x)\left(u^{\prime} \otimes v^{\prime}\right)\right) & =\theta\left(Y(u, x) u^{\prime} \otimes v^{\prime}\right) \\
& =\lim _{x_{2} \rightarrow 0} Y\left(\psi\left(Y(u, x) u^{\prime}\right), x_{2}\right) \phi\left(v^{\prime}\right) \\
& =\lim _{x_{2} \rightarrow 0} Y\left(Y(\psi(u), x) \psi\left(u^{\prime}\right), x_{2}\right) \phi\left(v^{\prime}\right) \\
& =\lim _{x_{2} \rightarrow 0} Y\left(\psi(u), x+x_{2}\right) Y\left(\psi\left(u^{\prime}\right), x_{2}\right) \phi\left(v^{\prime}\right) \\
& =Y(\psi(u), x) \theta\left(u^{\prime} \otimes v^{\prime}\right)
\end{aligned}
$$


and

$$
\begin{aligned}
\theta\left(Y_{\sharp}(\mathbf{1} \otimes v, x)\left(u^{\prime} \otimes v^{\prime}\right)\right) & =\theta\left(g u^{\prime} \otimes Y(v, x) v^{\prime}\right) \\
& =\lim _{x_{2} \rightarrow 0} Y\left(\psi\left(g u^{\prime}\right), x_{2}\right) \phi\left(Y(v, x) v^{\prime}\right) \\
& =\lim _{x_{2} \rightarrow 0} Y\left(\psi\left(g u^{\prime}\right), x_{2}\right) Y(\phi(v), x) \phi\left(v^{\prime}\right) \\
& =\lim _{x_{2} \rightarrow 0} Y\left(\phi(v), x+x_{2}\right) Y\left(\psi\left(u^{\prime}\right), x_{2}\right) \phi\left(v^{\prime}\right) \\
& =Y(\phi(v), x) \theta\left(u^{\prime} \otimes v^{\prime}\right) .
\end{aligned}
$$

As $U$ and $V$ generate $U \sharp_{G} V$, it follows that $\theta$ is a homomorphism.

We continue to study $U \sharp_{G} V$-modules.

Proposition 2.16. Let $E$ be a $U$-module on which $G$ acts such that

$$
g(Y(u, x) w)=Y(g u, x) g w \quad \text { for } g \in G, u \in U, w \in E,
$$

and let $F$ be any $V$-module. Then $E \otimes F$ is a $U \sharp_{G} V$-module with

$$
Y(u \otimes v, x)(w \otimes f)=Y(u, x) g w \otimes Y(v, x) f
$$

for $u \in U, v \in V[g], g \in G, w \in E, f \in F$. We denote this module by $E \sharp F$. Furthermore, if the $U$-module $E$ and the $V$-module $F$ are irreducible and if $E$ is of countable dimension (over $\mathbb{C}$ ), then $E \sharp F$ is an irreducible $U \sharp_{G} V$-module.

Proof. From the first part of the proof of Proposition 2.14, we see that this indeed defines a $U \sharp_{G} V$-module structure on $E \otimes F$. For proving irreducibility, let $W$ be any nonzero submodule of $E \otimes F$. Notice that $U$ acts on the first factor of $E \otimes F$. Since $E$ is an irreducible $U$-module of countable dimension (over $\mathbb{C}$ ), the Schur lemma holds and $\operatorname{End}_{U} E=\mathbb{C}$. Using Jacobson's density theorem on $E$, we get $W=E \otimes K$ for some subspace $K$ of $F$. From the definition of the action of $V$ on $E \otimes F$, we see that $K$ is a $V$-submodule of $F$. With $F$ an irreducible $V$-module we have $K=F$, proving $W=E \otimes F$. Thus $E \otimes F$ is an irreducible $U \sharp_{G} V$-module.

Corollary 2.17. Assume that both $U$ and $V$ are irreducible nonlocal vertex algebras and that $U$ is of countable dimension (over $\mathbb{C}$ ). Then $U \sharp_{G} V$ is an irreducible nonlocal vertex algebra.

Proposition 2.18. Let $W$ be a $U \sharp_{G} V$-module. Assume that $W$ contains an irreducible $U$-submodule E of countable dimension (over $\mathbb{C}$ ) on which $G$ acts such that

$$
g(Y(u, x) w)=Y(g u, x) g w \quad \text { for } g \in G, u \in U, w \in E
$$

and assume that $W$ is generated by $E$. Then $W$ is isomorphic to $E \otimes F$ for some $V$-module $F$. Furthermore, if $W$ is irreducible, then $F$ is an irreducible $V$-module. 
Proof. First we prove that $W$ as a $U$-module is a direct sum of irreducible modules isomorphic to $E$. Let $v \in V[g], n \in \mathbb{Z}$ with $g \in G$. It is clear that $v_{n} E$ is a $U$-submodule of $W$ and the linear map from $E$ to $v_{n} E$, sending $w$ to $v_{n} g^{-1} w$, is a $U$-homomorphism. Thus $v_{n} E$, if not zero, is an irreducible $U$-submodule of $W$, isomorphic to $E$. Let $W^{\prime}$ be the sum of the $U$-submodules $v_{n} E$ for $v \in V[g], n \in \mathbb{Z}$ with $g \in G$. It follows from Lemma 2.3 that $W^{\prime}$ is a $V$-submodule of $W$. As $U$ and $V$ generate $U \sharp_{G} V$, it follows that $W^{\prime}$ is a $U \sharp_{G} V$-submodule. Thus $W^{\prime}=W$ as $W$ is generated by $E$. This proves that $W$ as a $U$-module is a direct sum of copies of $E$.

With $E$ an irreducible $U$-module of countable dimension (over $\mathbb{C}$ ), we have $W=$ $E \otimes \operatorname{Hom}_{U}(E, W)$. For $v \in V[g], g \in G, f \in \operatorname{Hom}_{U}(E, W)$, we define $Y(v, x) f \in$ $\left(\operatorname{Hom}_{\mathbb{C}}(E, W)\right)\left[\left[x, x^{-1}\right]\right]$ by

$$
(Y(v, x) f)(w)=Y(v, x) f\left(g^{-1} w\right) \quad \text { for } w \in E .
$$

For $u \in U, w \in W$, we have

$$
\begin{aligned}
(Y(v, x) f)\left(Y\left(u, x_{0}\right) w\right) & =Y(v, x) f\left(g^{-1} Y\left(u, x_{0}\right) w\right) \\
& =Y(v, x) f\left(Y\left(g^{-1} u, x_{0}\right) g^{-1} w\right) \\
& =Y(v, x) Y\left(g^{-1} u, x_{0}\right) f\left(g^{-1} w\right) \\
& =Y\left(u, x_{0}\right) Y(v, x) f\left(g^{-1} w\right) \\
& =Y\left(u, x_{0}\right)(Y(v, x) f)(w) .
\end{aligned}
$$

This proves that $Y(v, x) f \in\left(\operatorname{Hom}_{U}(E, W)\right)\left[\left[x, x^{-1}\right]\right]$. Let $0 \neq w \in E$ be arbitrarily fixed. Since $E$ is an irreducible $U$-module, $g^{-1} w$ generates $E$. Let $l$ be a nonnegative integer such that $x^{l} Y(v, x) f\left(g^{-1} w\right) \in W[[x]]$. It follows that

$$
x^{l}(Y(v, x) f) \in\left(\operatorname{Hom}_{U}(E, W)\right)[[x]] .
$$

That is, $Y(v, x) f \in\left(\operatorname{Hom}_{U}(E, W)\right)((x))$. Furthermore, for $v \in V[g], v^{\prime} \in V[h]$ with $g, h \in G$ and for $w \in W$, we have

$\left(Y\left(v, x_{1}\right) Y\left(v^{\prime}, x_{2}\right) f\right)(w)=Y\left(v, x_{1}\right)\left(Y\left(v^{\prime}, x_{2}\right) f\right)\left(g^{-1} w\right)=Y\left(v, x_{1}\right) Y\left(v^{\prime}, x_{2}\right) f\left(h^{-1} g^{-1} w\right)$

and

$$
\left(Y\left(Y\left(v, x_{0}\right) v^{\prime}, x_{2}\right) f\right)(w)=Y\left(Y\left(v, x_{0}\right) v^{\prime}, x_{2}\right) f\left((g h)^{-1} w\right) .
$$

Now weak associativity follows. This proves that we have a $V$-module structure on $\operatorname{Hom}_{U}(E, W)$, so that $E \otimes \operatorname{Hom}_{U}(E, W)$ is a $U \sharp_{G} V$-module by Proposition 2.16 and we have $W=E \otimes \operatorname{Hom}_{U}(E, W)$ as a $U \sharp_{G} V$-module. If $W$ is irreducible, $\operatorname{Hom}_{U}(E, W)$ must be an irreducible $V$-module.

\section{Associative algebras $\mathcal{A}_{\mathrm{Q}}$ and quantum vertex al- gebras $V_{\mathrm{Q}}$}

In this section we associate an associative algebra $\mathcal{A}_{\mathbf{Q}}$ to each "skew" complex matrix $\mathbf{Q}$ and we study its modules, including vacuum modules. We show that on 
the universal vacuum module $V_{\mathbf{Q}}$ there exists a canonical irreducible quantum vertex algebra structure.

Definition 3.1. Let $l$ be a positive integer and let $\mathbf{Q}=\left(q_{i j}\right)_{i, j=1}^{l}$ be a complex matrix such that

$$
q_{i j} q_{j i}=1 \quad \text { for } 1 \leq i, j \leq l \text {. }
$$

Define $\mathcal{A}_{\mathbf{Q}}$ to be the associative algebra with identity (over $\mathbb{C}$ ) with generators

$$
X_{i, n}, Y_{i, n} \quad(i=1, \ldots, l, n \in \mathbb{Z}),
$$

subject to relations

$$
\begin{aligned}
& X_{i, m} X_{j, n}=q_{i j} X_{j, n} X_{i, m}, \quad Y_{i, m} Y_{j, n}=q_{i j} Y_{j, n} Y_{i, m} \\
& X_{i, m} Y_{j, n}-q_{j i} Y_{j, n} X_{i, m}=\delta_{i, j} \delta_{m+n+1,0}
\end{aligned}
$$

for $i, j=1, \ldots, l, m, n \in \mathbb{Z}$.

Let $\left\{e_{1}, \ldots, e_{l}\right\}$ denote the standard $\mathbb{Z}$-basis of $\mathbb{Z}^{l}$. It is straightforward to see that $\mathcal{A}_{\mathbf{Q}}$ is a $\mathbb{Z}^{l}$-graded algebra with the grading defined by

$$
\operatorname{deg} X_{i, m}=e_{i}, \quad \operatorname{deg} Y_{i, m}=-e_{i} \quad \text { for } 1 \leq i \leq l, m \in \mathbb{Z}
$$

Set

$$
\begin{aligned}
& \mathcal{A}_{\mathbf{Q}}^{+}=\left\langle X_{i, m}, Y_{j, n} \mid i, j=1, \ldots, l, m, n \geq 0\right\rangle, \\
& \mathcal{A}_{\mathbf{Q}}^{-}=\left\langle X_{i, m}, Y_{j, n} \mid i, j=1, \ldots, l, m, n<0\right\rangle,
\end{aligned}
$$

which are $\mathbb{Z}^{l}$-graded subalgebras of $\mathcal{A}_{\mathbf{Q}}$.

Notice that if $q_{i j}=1$ for all $i, j=1, \ldots, l$, the algebra $\mathcal{A}_{\mathbf{Q}}$, which is isomorphic to the universal enveloping algebra of an infinite-dimensional Heisenberg Lie algebra, is a Weyl algebra, and that if $q_{i j}=-1$ for all $i, j=1, \ldots, l, \mathcal{A}_{\mathbf{Q}}$ is a Clifford algebra. In general, we have $q_{i i}= \pm 1$ for $1 \leq i \leq l$ as $q_{i i} q_{i i}=1$. Then, for each $1 \leq i \leq l$, the algebra $\mathcal{A}_{\left(q_{i i}\right)}$ (associated with the $1 \times 1$ matrix $q_{i i}$ ) is either a Weyl algebra or a Clifford algebra. In the following we shall prove that $\mathcal{A}_{\mathbf{Q}}$ is isomorphic to a certain cocycle-twist of the tensor product algebra $\mathcal{A}_{\left(q_{11}\right)} \otimes \cdots \otimes \mathcal{A}_{\left(q_{l l}\right)}$.

For $1 \leq i \leq l$, we temporarily denote the generators of $\mathcal{A}_{\left(q_{i i}\right)}$ by $\bar{X}_{i, m}$ and $\bar{Y}_{i, m}$ for $m \in \mathbb{Z}$. Define a $\mathbb{Z}$-grading on $\mathcal{A}_{\left(q_{i i}\right)}$ by

$$
\operatorname{deg} \bar{X}_{i, m}=1, \quad \operatorname{deg} \bar{Y}_{i, m}=-1 \quad \text { for } m \in \mathbb{Z},
$$

making $\mathcal{A}_{\left(q_{i i}\right)}$ a $\mathbb{Z}$-graded algebra, where we denote by $\mathcal{A}_{\left(q_{i i}\right)}[n]$ the degree- $n$ subspace for $n \in \mathbb{Z}$. Set

$$
\mathcal{A}=\mathcal{A}_{\left(q_{11}\right)} \otimes \mathcal{A}_{\left(q_{22}\right)} \otimes \cdots \otimes \mathcal{A}_{\left(q_{l l}\right)}
$$


the tensor product algebra, which is naturally a $\mathbb{Z}^{l}$-graded algebra. We define a group homomorphism $\varepsilon: \mathbb{Z}^{l} \times \mathbb{Z}^{l} \rightarrow \mathbb{C}^{\times}$by

$$
\varepsilon\left(e_{i}, e_{j}\right)= \begin{cases}q_{i j} & \text { if } i>j \\ 1 & \text { if } i \leq j\end{cases}
$$

for $1 \leq i, j \leq l$, recalling that $\left\{e_{1}, \ldots, e_{l}\right\}$ is the standard $\mathbb{Z}$-basis of $\mathbb{Z}^{l}$. In particular, $\varepsilon$ is a normalized 2-cocycle of $\mathbb{Z}^{l}$. Denote by $\mathcal{A}^{\varepsilon}$ the $\varepsilon$-twist of $\mathcal{A}$. That is, $\mathcal{A}^{\varepsilon}=\mathcal{A}$ as a vector space and for $a \in \mathcal{A}[\alpha], b \in \mathcal{A}[\beta]$ with $\alpha, \beta \in \mathbb{Z}^{l}$,

$$
a^{\varepsilon} b^{\varepsilon}=\varepsilon(\alpha, \beta)(a b)^{\varepsilon},
$$

where for any $u \in \mathcal{A}$, we use $u^{\varepsilon}$ for $u$ viewed as an element of $\mathcal{A}^{\varepsilon}$. Notice that for each $1 \leq i \leq l$, as $\varepsilon\left(e_{i}, e_{i}\right)=1, \mathcal{A}_{\left(q_{i i}\right)}^{\varepsilon}=\mathcal{A}_{\left(q_{i i}\right)}$ as an algebra.

Proposition 3.2. The map

$$
\phi:\left\{\bar{X}_{i, m}^{\epsilon}, \bar{Y}_{i, m}^{\epsilon} \mid 1 \leq i \leq l, m \in \mathbb{Z}\right\} \subset \mathcal{A}^{\varepsilon} \rightarrow \mathcal{A}_{\mathbf{Q}} ; \quad \bar{X}_{i, m}^{\epsilon} \mapsto X_{i, m}, \quad \bar{Y}_{i, m}^{\epsilon} \mapsto Y_{i, m}
$$

extends to an algebra isomorphism from the $\varepsilon$-twist $\mathcal{A}^{\varepsilon}$ of the $\mathbb{Z}^{l}$-graded algebra $\mathcal{A}$ to $\mathcal{A}_{\mathbf{Q}}$. Furthermore, for each $1 \leq i \leq l$, the assignment

$$
\bar{X}_{i, m} \mapsto X_{i, m}, \quad \bar{Y}_{i, m} \mapsto Y_{i, m} \quad \text { for } m \in \mathbb{Z}
$$

gives rise to an algebra embedding of $\mathcal{A}_{\left(q_{i i}\right)}$ into $\mathcal{A}_{\mathbf{Q}}$, and the linear map

$$
\pi: \mathcal{A}_{\left(q_{11}\right)} \otimes \cdots \otimes \mathcal{A}_{\left(q_{l l}\right)} \rightarrow \mathcal{A}_{\mathbf{Q}} ; \quad\left(a_{1}, \ldots, a_{l}\right) \mapsto a_{1} \cdots a_{l}
$$

is a linear isomorphism preserving the $\mathbb{Z}^{l}$-gradings.

Proof. From the definition of $\varepsilon$ we get

$$
\varepsilon\left(e_{i}, e_{j}\right) \varepsilon\left(e_{j}, e_{i}\right)^{-1}=q_{i j} \quad \text { for } i \neq j .
$$

For $1 \leq i \neq j \leq l, m, n \in \mathbb{Z}$, we have

$$
\begin{aligned}
& \bar{X}_{i, m}^{\varepsilon} \bar{X}_{j, n}^{\varepsilon}=\varepsilon\left(e_{i}, e_{j}\right)\left(\bar{X}_{i, m} \bar{X}_{j, n}\right)^{\varepsilon}=\varepsilon\left(e_{i}, e_{j}\right) \varepsilon\left(e_{j}, e_{i}\right)^{-1} \bar{X}_{j, n}^{\varepsilon} \bar{X}_{i, m}^{\varepsilon}=q_{i j} \bar{X}_{j, n}^{\varepsilon} \bar{X}_{i, m}^{\varepsilon}, \\
& \bar{Y}_{i, m}^{\varepsilon} \bar{Y}_{j, n}^{\varepsilon}=q_{i j} \bar{Y}_{j, n}^{\varepsilon} \bar{Y}_{i, m}^{\varepsilon}, \\
& \bar{X}_{i, m}^{\varepsilon} \bar{Y}_{j, n}^{\varepsilon}=\varepsilon\left(e_{i},-e_{j}\right) \varepsilon\left(-e_{j}, e_{i}\right)^{-1} \bar{Y}_{j, n}^{\varepsilon} \bar{X}_{i, m}^{\varepsilon}=q_{i j}^{-1} \bar{Y}_{j, n}^{\varepsilon} \bar{X}_{i, m}^{\varepsilon}=q_{j i} \bar{Y}_{j, n}^{\varepsilon} \bar{X}_{i, m}^{\varepsilon} .
\end{aligned}
$$

Since $\varepsilon\left(e_{i}, e_{i}\right)=1$, we also have

$$
\begin{aligned}
& \bar{X}_{i, m}^{\varepsilon} \bar{X}_{i, n}^{\varepsilon}=\left(\bar{X}_{i, n} \bar{X}_{i, m}\right)^{\varepsilon}=q_{i i}\left(\bar{X}_{i, m} \bar{X}_{i, n}\right)^{\varepsilon}=q_{i i} \bar{X}_{i, n}^{\varepsilon} \bar{X}_{i, m}^{\varepsilon} \\
& \bar{Y}_{i, m}^{\varepsilon} \bar{Y}_{i, n}^{\varepsilon}=q_{i i} \bar{Y}_{i, n}^{\varepsilon} \bar{Y}_{i, m}^{\varepsilon}, \\
& \bar{X}_{i, m}^{\varepsilon} \bar{Y}_{i, n}^{\varepsilon}-q_{i i} \bar{Y}_{i, n}^{\varepsilon} \bar{X}_{i, m}^{\varepsilon}=\left(\bar{X}_{i, m} \bar{Y}_{i, n}\right)^{\varepsilon}-q_{i i}\left(\bar{Y}_{i, n} \bar{X}_{i, m}\right)^{\varepsilon}=\delta_{m+n+1,0}
\end{aligned}
$$


It follows that there exists an algebra homomorphism $\psi$ from $\mathcal{A}_{\mathbf{Q}}$ onto $\mathcal{A}^{\varepsilon}$ such that

$$
\psi\left(X_{i, m}\right)=\bar{X}_{i, m}^{\varepsilon}, \quad \psi\left(Y_{i, m}\right)=\bar{Y}_{i, m}^{\varepsilon} \quad \text { for } i=1, \ldots, l, m \in \mathbb{Z} .
$$

On the other hand, define a group homomorphism $\varepsilon^{\prime}: \mathbb{Z}^{l} \times \mathbb{Z}^{l} \rightarrow \mathbb{C}^{\times}$by

$$
\varepsilon^{\prime}\left(e_{i}, e_{j}\right)=\varepsilon\left(e_{i}, e_{j}\right)^{-1} \quad \text { for } 1 \leq i, j \leq l .
$$

We then consider the $\varepsilon^{\prime}$-twist $\mathcal{A}_{\mathbf{Q}}^{\varepsilon^{\prime}}$ of $\mathcal{A}_{\mathbf{Q}}$. It is straightforward to show that for $1 \leq i \neq j \leq l, m, n \in \mathbb{Z}$,

$$
\begin{aligned}
& X_{i, m}^{\varepsilon^{\prime}} X_{j, n}^{\varepsilon^{\prime}}=X_{j, n}^{\varepsilon^{\prime}} X_{i, m}^{\varepsilon^{\prime}}, \quad Y_{i, m}^{\varepsilon^{\prime}} Y_{j, n}^{\varepsilon^{\prime}}=Y_{j, n}^{\varepsilon^{\prime}} Y_{i, m}^{\varepsilon^{\prime}}, \quad X_{i, m}^{\varepsilon^{\prime}} Y_{j, n}^{\varepsilon^{\prime}}=Y_{j, n}^{\varepsilon^{\prime}} X_{i, m}^{\varepsilon^{\prime}}, \\
& X_{i, m}^{\varepsilon^{\prime}} X_{i, n}^{\varepsilon^{\prime}}=q_{i i} X_{i, n}^{\varepsilon^{\prime}} X_{i, m}^{\varepsilon^{\prime}}, \quad Y_{i, m}^{\varepsilon^{\prime}} Y_{i, n}^{\varepsilon^{\prime}}=q_{i i} Y_{i, n}^{\varepsilon^{\prime}} Y_{i, m}^{\varepsilon^{\prime}}, \quad X_{i, m}^{\varepsilon^{\prime}} Y_{i, n}^{\varepsilon^{\prime}}-q_{i i} Y_{i, n}^{\varepsilon^{\prime}} X_{i, m}^{\varepsilon^{\prime}}=\delta_{m+n+1,0} .
\end{aligned}
$$

It follows that there exists an algebra homomorphism from $\mathcal{A}$ to $\mathcal{A}_{\mathrm{Q}}^{\varepsilon^{\prime}}$, sending $\bar{X}_{i, m}$ to $X_{i, m}^{\varepsilon^{\prime}}$ and $\bar{Y}_{i, m}$ to $Y_{i, m}^{\varepsilon^{\prime}}$ for $1 \leq i \leq l, m \in \mathbb{Z}$. Furthermore, there exists an algebra homomorphism from $\mathcal{A}^{\varepsilon}$ to $\left(\mathcal{A}_{\mathbf{Q}}^{\varepsilon^{\prime}}\right)^{\varepsilon}=\mathcal{A}_{\mathbf{Q}}$, sending $\bar{X}_{i, m}^{\varepsilon}$ to $X_{i, m}$ and $\bar{Y}_{i, m}^{\varepsilon}$ to $Y_{i, m}$ for $1 \leq i \leq l, m \in \mathbb{Z}$. Now it follows that $\psi$ is an isomorphism from $\mathcal{A}_{\mathbf{Q}}$ onto $\mathcal{A}^{\varepsilon}$. Notice that for homogeneous $a_{1} \in \mathcal{A}_{\left(q_{11}\right)}\left[m_{1}\right], \ldots, a_{l} \in \mathcal{A}_{\left(q_{l l}\right)}\left[m_{l}\right]$, we have

$$
a_{1}^{\varepsilon} \cdots a_{l}^{\varepsilon}=\lambda\left(a_{1} \cdots a_{l}\right)^{\varepsilon}
$$

in $\mathcal{A}^{\varepsilon}$ for some nonzero number $\lambda$ depending on $m_{1}, \ldots, m_{l}$. Then all the assertions follow immediately.

Furthermore we have:

Corollary 3.3. The linear maps

$$
\begin{aligned}
\mathcal{A}_{\left(q_{11}\right)}^{ \pm} \otimes \cdots \otimes \mathcal{A}_{\left(q_{l l}\right)}^{ \pm} & \rightarrow \mathcal{A}_{\mathrm{Q}}^{ \pm} \\
a_{1} \otimes \cdots \otimes a_{l} & \mapsto a_{1} \cdots a_{l}
\end{aligned}
$$

are linear isomorphisms. Furthermore, the linear map

$$
\mathcal{A}_{\mathbf{Q}}^{-} \otimes \mathcal{A}_{\mathbf{Q}}^{+} \rightarrow \mathcal{A}_{\mathbf{Q}} ; \quad a \otimes b \mapsto a b
$$

is a linear isomorphism.

Proof. For each $1 \leq i \leq l$, it is well known that the linear map

$$
\mathcal{A}_{\left(q_{i i}\right)}^{-} \otimes \mathcal{A}_{\left(q_{i i}\right)}^{+} \rightarrow \mathcal{A}_{\left(q_{i i}\right)} ; a \otimes b \mapsto a b
$$

is a linear isomorphism. With $\mathcal{A}=\mathcal{A}_{\left(q_{11}\right)} \otimes \cdots \otimes \mathcal{A}_{\left(q_{l l}\right)}$, the linear map

$$
\mathcal{A}^{-} \otimes \mathcal{A}^{+} \rightarrow \mathcal{A} ; \quad a \otimes b \mapsto a b
$$


is a linear isomorphism, where

$$
\mathcal{A}^{ \pm}=\mathcal{A}_{\left(q_{11}\right)}^{ \pm} \otimes \cdots \otimes \mathcal{A}_{\left(q_{l l}\right)}^{ \pm}
$$

Clearly, $\mathcal{A}^{ \pm}$are $\mathbb{Z}^{l}$-graded subalgebras. For any homogeneous vectors $a_{1}, \ldots, a_{k} \in \mathcal{A}$, we have

$$
a_{1}^{\varepsilon} \cdots a_{k}^{\varepsilon}=\mu\left(a_{1} \cdots a_{k}\right)^{\varepsilon}
$$

where $\mu$ is a nonzero number depending only on the gradings of $a_{1}, \ldots, a_{k}$. It follows that $\mathcal{A}_{\mathbf{Q}}^{ \pm}=\left(\mathcal{A}^{ \pm}\right)^{\varepsilon}$, under the identification of $\mathcal{A}_{\mathbf{Q}}$ with $\mathcal{A}^{\varepsilon}$ in Proposition 3.2. Then all the assertions follow from the second assertion of Proposition 3.2 .

In the following we shall show that $\mathcal{A}_{\mathrm{Q}}$ can also be constructed inductively by using smash products of algebras $\mathcal{A}_{\left(q_{11}\right)}, \ldots, \mathcal{A}_{\left(q_{l l}\right)}$. First we formulate the following straightforward consequence of the definition:

Lemma 3.4. For any $\mathbf{q}=\left(q_{1}, \ldots, q_{l}\right) \in\left(\mathbb{C}^{\times}\right)^{l}$, there exists a (unique) automorphism $\sigma_{\mathbf{q}}$ of $\mathcal{A}_{\mathbf{Q}}$ such that

$$
\sigma_{\mathbf{q}}\left(X_{i, m}\right)=q_{i} X_{i, m}, \quad \sigma_{\mathbf{q}}\left(Y_{i, m}\right)=q_{i}^{-1} Y_{i, m}
$$

for $i=1, \ldots, l, m \in \mathbb{Z}$. Furthermore, the multiplicative group $\left(\mathbb{C}^{\times}\right)^{l}$ acts on $\mathcal{A}_{\mathbf{Q}}$ with $\mathbf{q}=\left(q_{1}, \ldots, q_{l}\right) \in\left(\mathbb{C}^{\times}\right)^{l}$ acting as $\sigma_{\mathbf{q}}$.

Note that any associative algebra with identity is a nonlocal vertex algebra. Then all the results for smash product nonlocal vertex algebras in Section 2 hold for the degenerate case with $U=A$ and $V=B$ classical associative algebras.

Proposition 3.5. Let $\mathbf{Q}=\left(q_{i j}\right)_{i, j=1}^{l}$ be a complex matrix with $l \geq 2$ such that $q_{i j} q_{j i}=1$ for $1 \leq i, j \leq l$. Set $\mathbf{Q}^{\prime}=\left(q_{i j}\right)_{1 \leq i, j \leq l-1}$ and set

$$
\mathbf{q}=\left(q_{1, l}, \ldots, q_{l-1, l}\right) \in\left(\mathbb{C}^{\times}\right)^{l-1} .
$$

Let $\mathbb{Z}$ act on $\mathcal{A}_{\mathbf{Q}^{\prime}}$ with $n$ acting as $\sigma_{\mathbf{q}}^{n}$ for $n \in \mathbb{Z}$ and equip $\mathcal{A}_{\left(q_{l l}\right)}$ with the $\mathbb{Z}$-grading constructed before. Then the natural algebra homomorphisms from $\mathcal{A}_{\mathrm{Q}^{\prime}}$ and from $\mathcal{A}_{\left(q_{l l}\right)}$ to $\mathcal{A}_{\mathbf{Q}}$ give rise to an algebra isomorphism from $\mathcal{A}_{\mathbf{Q}^{\prime}} \sharp_{\mathbb{Z}} \mathcal{A}_{\left(q_{l l}\right)}$ to $\mathcal{A}_{\mathbf{Q}}$.

Proof. Let $X_{m}, Y_{m}(m \in \mathbb{Z})$ denote the generators of $\mathcal{A}_{\left(q_{l l}\right)}$. For $1 \leq i, j \leq l-1, m \in$ $\mathbb{Z}$, set $\tilde{X}_{i, m}=X_{i, m}, \tilde{Y}_{i, m}=Y_{i, m}$, and $\tilde{X}_{l, m}=X_{m}, \tilde{Y}_{l, m}=Y_{m}$, which are elements of $\mathcal{A}_{\mathbf{Q}^{\prime} \sharp_{\mathbb{Z}}} \mathcal{A}_{\left(q_{l l}\right)}$. For $1 \leq i \leq l-1, m, n \in \mathbb{Z}$, noticing that $\operatorname{deg} X_{m}=-1$ and $\operatorname{deg} Y_{n}=1$, we have

$$
\begin{aligned}
& \tilde{X}_{l, n} \tilde{X}_{i, m}=X_{n} X_{i, m}=\sigma_{\mathbf{q}}^{-1}\left(X_{i, m}\right) X_{n}=q_{i, l}^{-1} X_{i, m} X_{n}=q_{l, i} \tilde{X}_{i, m} \tilde{X}_{l, n} \\
& \tilde{Y}_{l, n} \tilde{Y}_{i, m}=Y_{n} Y_{i, m}=\sigma_{\mathbf{q}}\left(Y_{i, m}\right) Y_{n}=q_{i, l}^{-1} Y_{i, m} Y_{n}=q_{l, i} \tilde{Y}_{i, m} \tilde{Y}_{l, n} \\
& \tilde{Y}_{l, n} \tilde{X}_{i, m}=Y_{n} X_{i, m}=\sigma_{\mathbf{q}}\left(X_{i, m}\right) Y_{n}=q_{i, l} X_{i, m} Y_{n}=q_{l, i}^{-1} \tilde{X}_{i, m} \tilde{Y}_{l, n} \\
& \tilde{X}_{l, n} \tilde{Y}_{i, m}=X_{n} Y_{i, m}=\sigma_{\mathbf{q}}^{-1}\left(Y_{i, m}\right) X_{n}=q_{i, l} Y_{i, m} X_{n}=q_{i, l} \tilde{Y}_{i, m} \tilde{X}_{l, n}
\end{aligned}
$$


It follows that there exists an algebra homomorphism $\pi$ from $\mathcal{A}_{\mathbf{Q}}$ to $\mathcal{A}_{\mathbf{Q}^{\prime} \mathbb{Z}_{\mathbb{Z}}} \mathcal{A}_{\left(q_{l l}\right)}$, sending $X_{i, m}$ to $\tilde{X}_{i, m}, Y_{i, m}$ to $\tilde{Y}_{i, m}$ for $1 \leq i, j \leq l, m \in \mathbb{Z}$. On the other hand, let $f$ be the natural algebra homomorphism from $\mathcal{A}_{\mathbf{Q}^{\prime}}$ to $\mathcal{A}_{\mathbf{Q}}$ and $g$ the natural algebra homomorphism from $\mathcal{A}_{\left(q_{l l}\right)}$ to $\mathcal{A}_{\mathbf{Q}}$. By Proposition 2.15 we have an algebra

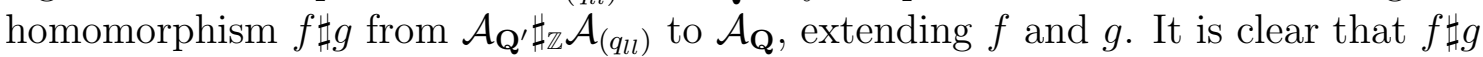
is inverse to $\pi$, so that $f \sharp g$ is an isomorphism.

Recall that $\mathcal{A}_{\mathbf{Q}}^{ \pm}$are the subalgebras of $\mathcal{A}_{\mathbf{Q}}$, generated by $X_{i, m}, Y_{i, m}$ for $1 \leq i \leq$ $l, m \geq 0$. A vector $w$ in an $\mathcal{A}_{\mathbf{Q}}$-module $W$ is called a vacuum vector if $\mathcal{A}_{\mathbf{Q}}^{+} w=0$, and an $\mathcal{A}_{\mathbf{Q}}$-module $W$ equipped with a vacuum vector which generates $W$ is called a vacuum $\mathcal{A}_{\mathbf{Q}}$-module.

Set

$$
V_{\mathbf{Q}}=\mathcal{A}_{\mathbf{Q}} /\left(\mathcal{A}_{\mathbf{Q}} \mathcal{A}_{\mathbf{Q}}^{+}\right),
$$

a left $\mathcal{A}_{\mathbf{Q}^{-} \text {-module, and set }}$

$$
\mathbf{1}=1+\left(\mathcal{A}_{\mathbf{Q}} \mathcal{A}_{\mathbf{Q}}^{+}\right) \in V_{\mathbf{Q}} .
$$

Clearly, $\mathbf{1}$ is a vacuum vector and $V_{\mathbf{Q}}$ equipped with $\mathbf{1}$ is a vacuum $\mathcal{A}_{\mathbf{Q}^{-}}$-module. Furthermore, $V_{\mathbf{Q}}$ is universal in the obvious sense.

Proposition 3.6. The vacuum $\mathcal{A}_{\mathbf{Q}}$-module $V_{\mathbf{Q}}$ is irreducible and every nonzero vacuum $\mathcal{A}_{\mathbf{Q}}$-module is irreducible and isomorphic to $V_{\mathbf{Q}}$.

Proof. Notice that if the universal vacuum module $V_{\mathbf{Q}}$ is irreducible, the other assertions follow immediately. We shall use induction on $l$ to show that $V_{\mathbf{Q}}$ is an irreducible $\mathcal{A}_{\mathbf{Q}}$-module. If $l=1, \mathcal{A}_{\mathbf{Q}}=\mathcal{A}_{\left(q_{11}\right)}$ is a Weyl algebra or a Clifford algebra and it is well known that $V_{\left(q_{11}\right)}$ is an irreducible $\mathcal{A}_{\left(q_{11}\right)}$-module. Now, assume that $l \geq 2$. For a given $l \times l$ matrix $\mathbf{Q}$, set $\mathbf{Q}^{\prime}=\left(q_{i j}\right)_{i, j=1}^{l-1}$. By the induction hypothesis $V_{\mathbf{Q}^{\prime}}$ is an irreducible $\mathcal{A}_{\mathrm{Q}^{\prime}}$-module and every nonzero vacuum $\mathcal{A}_{\mathrm{Q}^{\prime}}$-module is isomorphic to

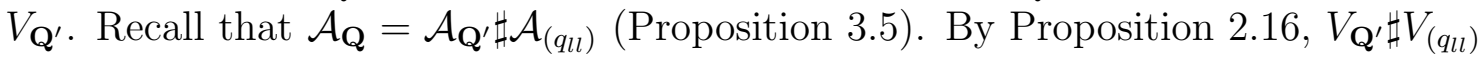

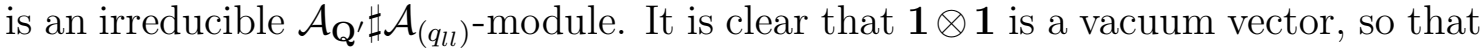
$V_{\mathbf{Q}^{\prime} \sharp V_{\left(q_{l l}\right)}}$ is a vacuum $\mathcal{A}_{\mathbf{Q}}$-module. Let $\psi$ denote the natural $\mathcal{A}_{\mathbf{Q}}$-homomorphism from $\mathcal{A}_{\mathbf{Q}}=\mathcal{A}_{\mathbf{Q}^{\prime} \sharp \mathcal{A}_{\left(q_{l l}\right)}}$ to $V_{\mathbf{Q}}$, sending $a \in \mathcal{A}_{\mathbf{Q}}$ to $a \mathbf{1}$. Let $a^{\prime} \in \mathcal{A}_{\mathbf{Q}^{\prime}}, b \in \mathcal{A}_{\left(q_{l l}\right)}[m]$ with $m \in \mathbb{Z}$. We have $\psi\left(a^{\prime} \sharp b\right)=a^{\prime} b \mathbf{1}=b \sigma_{\mathbf{q}}^{-m}\left(a^{\prime}\right) \mathbf{1}$. As $\sigma_{\mathbf{q}}\left(\mathcal{A}_{\mathbf{Q}^{\prime}}^{+}\right) \subset \mathcal{A}_{\mathbf{Q}^{\prime}}^{+}$, we see that $\psi$ gives rise to an $\mathcal{A}_{\mathbf{Q}^{-}}$-homomorphism $\bar{\psi}$ from $V_{\mathbf{Q}^{\prime} \sharp V_{\left(q_{l l}\right)}}$ to $V_{\mathbf{Q}}$, sending $\mathbf{1} \otimes \mathbf{1}$ to $\mathbf{1}$. As $V_{\mathbf{Q}^{\prime} \sharp V_{\left(q_{l l}\right)}}$ is irreducible, it follows that $\bar{\psi}$ is an isomorphism. Consequently, $V_{\mathbf{Q}}$ is an irreducible $\mathcal{A}_{\mathbf{Q}^{-}}$module.

For $1 \leq i \leq l$, set

$$
u^{(i)}=X_{i,-1} \mathbf{1}, \quad v^{(i)}=Y_{i,-1} \mathbf{1} \in V_{\mathbf{Q}}
$$

and set

$$
X_{i}(x)=\sum_{n \in \mathbb{Z}} X_{i, n} x^{-n-1}, \quad Y_{i}(x)=\sum_{n \in \mathbb{Z}} Y_{i, n} x^{-n-1} \in \mathcal{A}_{\mathbf{Q}}\left[\left[x, x^{-1}\right]\right] .
$$


Theorem 3.7. Let $\mathbf{Q}=\left(q_{i j}\right)_{1 \leq i, j \leq l}$ be a complex matrix such that $q_{i j} q_{j i}=1$ for $1 \leq i, j \leq l$, let $\mathcal{A}_{\mathbf{Q}}$ be the associative algebra associated with $\mathbf{Q}$ and let $V_{\mathbf{Q}}$ be the universal vacuum $\mathcal{A}_{\mathrm{Q}}$-module. There exists a (unique) irreducible quantum vertex algebra structure on $V_{\mathbf{Q}}$ with $\mathbf{1}$ as the vacuum vector such that

$$
Y\left(u^{(i)}, x\right)=X_{i}(x), \quad Y\left(v^{(i)}, x\right)=Y_{i}(x) \quad \text { for } i=1, \ldots, l .
$$

Let $W$ be any $\mathcal{A}_{\mathbf{Q}}$-module satisfying the condition that for any $w \in W, X_{i, m} w=$ $Y_{i, m} w=0$ for $1 \leq i \leq l$ and for $m$ sufficiently large. Then there exists a (unique) $V_{\mathbf{Q}}$-module structure on $W$ with

$$
Y_{W}\left(u^{(i)}, x\right)=X_{i}(x), \quad Y_{W}\left(v^{(i)}, x\right)=Y_{i}(x) \quad \text { for } i=1, \ldots, l .
$$

Conversely, any $V_{\mathbf{Q}^{-}}$-module $W$ is an $\mathcal{A}_{\mathbf{Q}^{-} \text {-module with }}$

$$
X_{i}(x)=Y_{W}\left(u^{(i)}, x\right), \quad Y_{i}(x)=Y_{W}\left(v^{(i)}, x\right) \quad \text { for } i=1, \ldots, l .
$$

Proof. For $m \in \mathbb{Z}, k \geq 1$, let

$$
Z_{m}, Z_{m}^{(1)}, \ldots, Z_{m}^{(k)} \in\left\{X_{i, m}, Y_{i, m} \mid 1 \leq i \leq l\right\}
$$

It follows from induction on $k$ that

$$
Z_{m} Z_{m_{1}}^{(1)} \cdots Z_{m_{k}}^{(k)} \mathbf{1}=0
$$

whenever $m \geq 0$ and $m \notin\left\{-m_{1}-1, \ldots,-m_{k}-1\right\}$. Then

$$
\left\{X_{i}(x), Y_{i}(x) \mid 1 \leq i \leq l\right\} \subset \mathcal{E}\left(V_{\mathbf{Q}}\right)=\operatorname{Hom}\left(V_{\mathbf{Q}}, V_{\mathbf{Q}}((x))\right) .
$$

Set $T=\left\{X_{i}(x), Y_{i}(x) \mid i=1, \ldots, l\right\}$. From the defining relations of $\mathcal{A}_{\mathbf{Q}}, T$ is an $\mathcal{S}$-local subset of $\mathcal{E}\left(V_{\mathbf{Q}}\right)$. By Theorem 5.8 of [Li6], $T$ generates a weak quantum vertex algebra $\langle T\rangle$, where the vertex operator map is denoted by $Y_{\mathcal{E}}$, with $V_{\mathbf{Q}}$ as a module where $Y_{V_{\mathbf{Q}}}\left(\alpha(x), x_{0}\right)=\alpha\left(x_{0}\right)$ for $\alpha(x) \in\langle T\rangle$. By Proposition 6.7 of [Li6], we have

$$
\begin{aligned}
& Y_{\mathcal{E}}\left(X_{i}(x), x_{1}\right) Y_{\mathcal{E}}\left(X_{j}(x), x_{2}\right)=q_{i j} Y_{\mathcal{E}}\left(X_{j}(x), x_{2}\right) Y_{\mathcal{E}}\left(X_{i}(x), x_{1}\right) \\
& Y_{\mathcal{E}}\left(Y_{i}(x), x_{1}\right) Y_{\mathcal{E}}\left(Y_{j}(x), x_{2}\right)=q_{i j} Y_{\mathcal{E}}\left(Y_{j}(x), x_{2}\right) Y_{\mathcal{E}}\left(Y_{i}(x), x_{1}\right), \\
& Y_{\mathcal{E}}\left(X_{i}(x), x_{1}\right) Y_{\mathcal{E}}\left(Y_{j}(x), x_{2}\right)-q_{j i} Y_{\mathcal{E}}\left(Y_{j}(x), x_{2}\right) Y_{\mathcal{E}}\left(X_{i}(x), x_{1}\right)=\delta_{i j} x_{2}^{-1} \delta\left(\frac{x_{1}}{x_{2}}\right)
\end{aligned}
$$

for $i, j=1, \ldots, l$. It follows that $\langle T\rangle$ is an $\mathcal{A}_{\mathbf{Q}}$-module with

$$
X_{i}\left(x_{0}\right)=Y_{\mathcal{E}}\left(X_{i}(x), x_{0}\right), \quad Y_{i}\left(x_{0}\right)=Y_{\mathcal{E}}\left(Y_{i}(x), x_{0}\right) \quad \text { for } 1 \leq i \leq l .
$$

It is clear that $\langle T\rangle$ is a vacuum module with $1_{V_{\mathbf{Q}}}$ as the vacuum vector. From the construction of $V_{\mathbf{Q}}$, there is an $\mathcal{A}_{\mathbf{Q}^{-}}$-module homomorphism $\pi$ from $V_{\mathbf{Q}}$ to $\langle T\rangle$, sending 1 to $1_{V_{\mathbf{Q}}}$. Now, in view of Theorem 6.3 of [Li6], there exists a weak quantum vertex 
algebra structure on $V_{\mathbf{Q}}$ with all the required properties. As $V_{\mathbf{Q}}$ is an irreducible $\mathcal{A}_{\mathbf{Q}^{-}}$ module, $V_{\mathbf{Q}}$ as a $V_{\mathbf{Q}^{-}}$-module is irreducible. Therefore, $V_{\mathbf{Q}}$ is an irreducible quantum vertex algebra.

Let $W$ be any $\mathcal{A}_{\mathbf{Q}^{-}}$-module satisfying the condition that for any $w \in W, X_{i, m} w=$ $Y_{i, m} w=0$ for $1 \leq i \leq l$ and for $m$ sufficiently large. Then $T_{W}=\left\{X_{i}(x), Y_{i}(x) \mid i=\right.$ $1, \ldots, l\}$, viewed as a subset of $\mathcal{E}(W)$, is $\mathcal{S}$-local and then by Theorem 5.8 of [Li6], $T_{W}$ generates a weak quantum vertex algebra $\left\langle T_{W}\right\rangle$ with $W$ as a module. It follows from the same argument that $\left\langle T_{W}\right\rangle$ is a vacuum $\mathcal{A}_{\mathrm{Q}^{-}}$-module and that there exists an $\mathcal{A}_{\mathbf{Q}^{-}}$ module homomorphism $\psi$ from $V_{\mathbf{Q}}$ to $\left\langle T_{W}\right\rangle$, sending 1 to $1_{W}$. As $V_{\mathbf{Q}}$ is generated by $u^{(i)}, v^{(i)}$, it follows that $\psi$ is a weak-quantum-vertex-algebra homomorphism. Consequently, $W$ is a $V_{\mathbf{Q}^{-}}$module.

On the other hand, let $\left(W, Y_{W}\right)$ be a $V_{\mathbf{Q}^{-}}$module. By Proposition 6.7 of [Li6], we have

$$
\begin{aligned}
& Y_{W}\left(u^{(i)}, x_{1}\right) Y_{W}\left(u^{(j)}, x_{2}\right)=q_{i j} Y_{W}\left(u^{(j)}, x_{2}\right) Y_{W}\left(u^{(i)}, x_{1}\right), \\
& Y_{W}\left(v^{(i)}, x_{1}\right) Y_{W}\left(v^{(j)}, x_{2}\right)=q_{i j} Y_{W}\left(v^{(j)}, x_{2}\right) Y_{W}\left(v^{(i)}, x_{1}\right), \\
& Y_{W}\left(u^{(i)}, x_{1}\right) Y_{W}\left(v^{(j)}, x_{2}\right)-q_{j i} Y_{W}\left(v^{(j)}, x_{2}\right) Y_{W}\left(u^{(i)}, x_{1}\right)=\delta_{i j} x_{2}^{-1} \delta\left(\frac{x_{1}}{x_{2}}\right)
\end{aligned}
$$

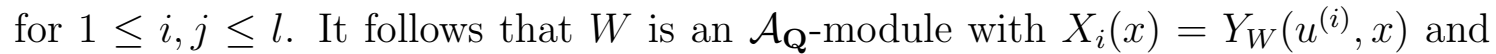
$Y_{i}(x)=Y_{W}\left(v^{(i)}, x\right)$ for $i=1, \ldots, l$. For any $w \in W$, as $Y_{W}(a, x) w \in W((x))$ for $a \in V_{\mathbf{Q}}$, we have $X_{i, m} w=Y_{i, m} w=0$ for $1 \leq i \leq l$ and for $m$ sufficiently large.

Furthermore, we have:

Proposition 3.8. Let $V$ be any nonlocal vertex algebra and let $\psi$ be any map from $\left\{u^{(i)}, v^{(i)} \mid i=1, \ldots, l\right\}$ to $V$ such that

$$
\begin{aligned}
& Y\left(\psi\left(u^{(i)}\right), x_{1}\right) Y\left(\psi\left(u^{(j)}\right), x_{2}\right)=q_{i j} Y\left(\psi\left(u^{(j)}\right), x_{2}\right) Y\left(\psi\left(u^{(i)}\right), x_{1}\right), \\
& Y\left(\psi\left(v^{(i)}\right), x_{1}\right) Y\left(\psi\left(v^{(j)}\right), x_{2}\right)=q_{i j} Y\left(\psi\left(v^{(j)}\right), x_{2}\right) Y\left(\psi\left(v^{(i)}\right), x_{1}\right), \\
& Y\left(\psi\left(u^{(i)}\right), x_{1}\right) Y\left(\psi\left(v^{(j)}\right), x_{2}\right)-q_{j i} Y\left(\psi\left(v^{(j)}\right), x_{2}\right) Y\left(\psi\left(u^{(i)}\right), x_{1}\right)=\delta_{i j} x_{2}^{-1} \delta\left(\frac{x_{1}}{x_{2}}\right)
\end{aligned}
$$

for $1 \leq i, j \leq l$. Then there exists a unique nonlocal-vertex-algebra homomorphism from $V_{\mathbf{Q}}$ to $V$, extending $\psi$.

Proof. The uniqueness is clear. From the assumption we see that $V$ is an $\mathcal{A}_{\mathrm{Q}^{-}}$ module with $X_{i}(x)=Y\left(\psi\left(u^{(i)}\right), x\right)$ and $Y_{i}(x)=Y\left(\psi\left(v^{(i)}\right), x\right)$ for $1 \leq i \leq l$, that the submodule generated by the vacuum vector is a vacuum $\mathcal{A}_{\mathbf{Q}}$-module. Then there exists an $\mathcal{A}_{\mathbf{Q}}$-module homomorphism $\tilde{\psi}$ from $V_{\mathbf{Q}}$ to $V$, sending the vacuum vector to the vacuum vector. As $V_{\mathbf{Q}}$ is generated by $u^{(i)}, v^{(i)}(i=1, \ldots, l)$, it follows that $\tilde{\psi}$ is a nonlocal-vertex-algebra homomorphism, extending $\psi$. 
Let $V$ be a nonlocal vertex algebra. Define $L C(V)$ to consist of vectors $a \in V$ satisfying the condition that for every $v \in V$, there exists a nonnegative integer $k$ such that

$$
\left(x_{1}-x_{2}\right)^{k}\left[Y\left(a, x_{1}\right), Y\left(v, x_{2}\right)\right]=0 .
$$

One can show that $L C(V)$ is a subalgebra of $V$ and it is a vertex algebra itself.

Definition 3.9. Let $V$ be a nonlocal vertex algebra. A vector $\omega \in V$ is called a conformal vector if $\omega \in L C(V)$ and if the following Virasoro algebra relation holds for $m, n \in \mathbb{Z}$,

$$
[L(m), L(n)]=(m-n) L(m+n)+\frac{1}{12}\left(m^{3}-m\right) \delta_{m+n, 0} c
$$

and

$$
[L(-1), Y(v, x)]=Y(L(-1) v, x)=\frac{d}{d x} Y(v, x)
$$

for all $v \in V$, where $Y(\omega, x)=\sum_{n \in \mathbb{Z}} L(n) x^{-n-2}$ and $c$ is a complex number. A nonlocal vertex algebra equipped with a conformal vector is called a conformal nonlocal vertex algebra.

Remark 3.10. Recall that for $\epsilon= \pm 1, \mathcal{A}_{(\epsilon)}$ is the associative algebra with generators $a_{m}, b_{n}$ for $m, n \in \mathbb{Z}$, subject to relations

$$
a_{m} a_{n}=\epsilon a_{n} a_{m}, \quad b_{m} b_{n}=\epsilon b_{n} b_{m}, \quad a_{m} b_{n}-\epsilon b_{n} a_{m}=\delta_{m+n+1,0} .
$$

Set $a=a_{-1} \mathbf{1}, b=b_{-1} \mathbf{1} \in V_{(\epsilon)}$. It has been known ([FFR], [W]; cf. [D1], [D2]) that there exists a (unique) conformal vertex superalgebra structure on $V_{(\epsilon)}$ with $\mathbf{1}$ as the vacuum vector and with

$$
Y(a, x)=\sum_{n \in \mathbb{Z}} a_{n} x^{-n-1} \quad \text { and } \quad Y(b, x)=\sum_{n \in \mathbb{Z}} b_{n} x^{-n-1},
$$

and with the conformal vector

$$
\omega=\frac{1}{2}\left(b_{-2} a-\epsilon a_{-2} b\right)
$$

of central charge $-\epsilon$. Furthermore, $V_{(\epsilon)}$ is simple and is $\frac{1}{2} \mathbb{Z}_{+}$-graded by the $L(0)$ weights with

$$
\left(V_{(\epsilon)}\right)_{(0)}=\mathbb{C} \mathbf{1} \text { and }\left(V_{(\epsilon)}\right)_{(1 / 2)}=\mathbb{C} a+\mathbb{C} b .
$$

On the other hand, $\mathcal{A}_{(\epsilon)}$ is a $\mathbb{Z}$-graded algebra with $\operatorname{deg} a_{m}=1$, $\operatorname{deg} b_{n}=-1$ for $m, n \in \mathbb{Z}$, and the vacuum module $V_{(\epsilon)}$ is naturally a $\mathbb{Z}$-graded $\mathcal{A}_{(\epsilon)}$-module with $\operatorname{deg} \mathbf{1}=0$. For $m, n \in \mathbb{Z}$, we have

$$
a_{m} V_{(\epsilon)}[n] \subset V_{(\epsilon)}[n+1], \quad b_{m} V_{(\epsilon)}[n] \subset V_{(\epsilon)}[n-1] .
$$

Since $V_{(\epsilon)}$ as a vertex superalgebra is generated by $a$ and $b$, it follows from induction that $V_{(\epsilon)}$ equipped with the $\mathbb{Z}$-grading is a vertex $\mathbb{Z}$-graded superalgebra with $\omega \in$ $\left(V_{(\epsilon)}\right)[0]$. 
Proposition 3.11. Let $\mathbf{Q}$ be an $l \times l$ complex matrix as before. Then $V_{\mathbf{Q}}$ is a conformal quantum vertex algebra of central charge $-\left(q_{11}+\cdots+q_{l l}\right)$ with conformal vector

$$
\omega=\frac{1}{2} \sum_{i=1}^{l}\left(v_{-2}^{(i)} u^{(i)}-q_{i i} u_{-2}^{(i)} v^{(i)}\right)
$$

and $V_{\mathbf{Q}}$ is $\frac{1}{2} \mathbb{Z}_{+}$-graded by the $L(0)$-weights such that $\left(V_{\mathbf{Q}}\right)_{(0)}=\mathbb{C} \mathbf{1}$ and

$$
\left(V_{\mathbf{Q}}\right)_{(1 / 2)}=\operatorname{span}\left\{u^{(i)}, v^{(i)} \mid 1 \leq i \leq l\right\} .
$$

Proof. We have simple conformal vertex superalgebras $V_{\left(q_{11}\right)}, \ldots, V_{\left(q_{l l}\right)}$. For $1 \leq i \leq$ $l$, denote the two generators of $V_{\left(q_{i i}\right)}$ by $a^{(i)}, b^{(i)}$. Set

$$
U=V_{\left(q_{11}\right)} \otimes \cdots \otimes V_{\left(q_{l l}\right)},
$$

the tensor product conformal vertex superalgebra with the conformal vector

$$
\omega=\frac{1}{2} \sum_{i=1}^{l}\left(b_{-2}^{(i)} a^{(i)}-q_{i i} a_{-2}^{(i)} b^{(i)}\right) .
$$

Then $U$ is $\frac{1}{2} \mathbb{Z}_{+}$-graded by the $L(0)$-weights with $U_{(0)}=\mathbb{C} \mathbf{1}$ and

$$
U_{(1 / 2)}=\operatorname{span}\left\{a^{(i)}, b^{(i)} \mid 1 \leq i \leq l\right\} .
$$

On the other hand, as $V_{\left(q_{11}\right)}, \ldots, V_{\left(q_{l l}\right)}$ are vertex $\mathbb{Z}$-graded superalgebras, $U$ is a vertex $\mathbb{Z}^{l}$-graded superalgebra. Let $\varepsilon: \mathbb{Z}^{l} \times \mathbb{Z}^{l} \rightarrow \mathbb{C}^{\times}$be the group homomorphism defined in (3.5). In view of Lemma 2.13, we have a nondegenerate quantum vertex algebra $\left(U^{\varepsilon}, Y_{\varepsilon}, \mathbf{1}\right)$. A straightforward calculation yields

$$
\begin{aligned}
& Y_{\varepsilon}\left(a^{(i)}, x_{1}\right) Y_{\varepsilon}\left(a^{(j)}, x_{2}\right)=q_{i j} Y_{\varepsilon}\left(a^{(j)}, x_{2}\right) Y_{\varepsilon}\left(a^{(i)}, x_{1}\right), \\
& Y_{\varepsilon}\left(b^{(i)}, x_{1}\right) Y_{\varepsilon}\left(b^{(j)}, x_{2}\right)=q_{i j} Y_{\varepsilon}\left(b^{(j)}, x_{2}\right) Y_{\varepsilon}\left(b^{(i)}, x_{1}\right), \\
& Y_{\varepsilon}\left(a^{(i)}, x_{1}\right) Y_{\varepsilon}\left(b^{(j)}, x_{2}\right)-q_{j i} Y_{\varepsilon}\left(b^{(j)}, x_{2}\right) Y_{\varepsilon}\left(a^{(i)}, x_{1}\right)=\delta_{i j} x_{2}^{-1} \delta\left(\frac{x_{1}}{x_{2}}\right)
\end{aligned}
$$

for $1 \leq i, j \leq l$. Hence, by Proposition 3.8, there is a nonlocal vertex algebra homomorphism $\psi$ from $V_{\mathbf{Q}}$ to $U^{\varepsilon}$ such that

$$
\psi\left(u_{i}\right)=\left(a^{(i)}\right)^{\varepsilon}, \quad \psi\left(v^{i}\right)=\left(b^{(i)}\right)^{\varepsilon} \quad \text { for } 1 \leq i \leq l .
$$

As $V_{\mathbf{Q}}$ is simple, $\psi$ is an isomorphism. Notice that $\omega \in U[\mathbf{0}]$, the degree-0 subspace of $U$ with respect to the $\mathbb{Z}^{l}$-grading. It follows that $\omega \in L C\left(U^{\varepsilon}\right)$ and $Y_{\varepsilon}(\omega, x)=$ $Y(\omega, x)$ on $U$. Then $U^{\varepsilon}$ equipped with the conformal vector $\omega$ is a conformal vertex algebra. 
Proposition 3.12. Let $W$ be a $V_{\mathbf{Q}}$-module satisfying the condition that for any $w \in W$, there exists a positive integer $k$ such that $\left(\mathcal{A}_{\mathbf{Q}}^{+}\right)^{k} w=0$. Then $W$ is a direct sum of irreducible modules isomorphic to the adjoint module $V_{\mathbf{Q}}$.

Proof. If $l=1, \mathcal{A}_{\mathbf{Q}}=\mathcal{A}_{\left(q_{11}\right)}$ is either a Weyl algebra or a Clifford algebra and it is well known that the assertion is true. If $l \geq 2$, by Proposition 3.5, $\mathcal{A}_{\mathbf{Q}}=\mathcal{A}_{\mathbf{Q}^{\prime} \sharp \mathcal{A}_{\left(q_{l l}\right)}}$ with $\mathbf{Q}^{\prime}=\left(q_{i j}\right)_{i, j=1}^{l-1}$. Assume that the assertion holds for $\mathcal{A}_{\mathbf{Q}^{\prime}}$. Let $W$ be any $\mathcal{A}_{\mathbf{Q}}$-module satisfying the condition that for any $w \in W$, there exists a positive integer $k$ such that $\left(\mathcal{A}_{\mathbf{Q}}^{+}\right)^{k} w=0$. Then $W$ as an $\mathcal{A}_{\mathbf{Q}^{\prime}}$-module is a direct sum of

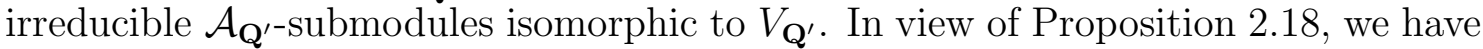
$W=V_{\mathbf{Q}^{\prime} \sharp F}$ for some $\mathcal{A}_{\left(q_{l l}\right)}$-module $F$. We know that $F$ is a direct sum of irreducible

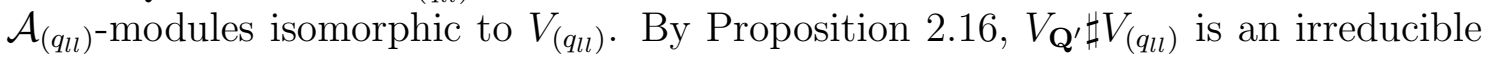

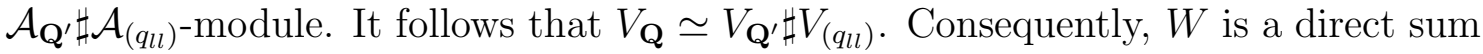
of irreducible modules isomorphic to $V_{\mathbf{Q}}$.

For the rest of this section, we establish certain results for $V_{\mathbf{Q}}$, which we shall need in the next section.

Definition 3.13. Let $V$ be a nonlocal vertex algebra. A pseudo-endomorphism of $V$ (see [Li5]) is a linear map $\Phi(x): V \rightarrow V \otimes \mathbb{C}((x))$ such that $\Phi(x)(\mathbf{1})=\mathbf{1}$ and

$$
\Phi\left(x_{1}\right) Y\left(v, x_{2}\right)=Y\left(\Phi\left(x_{1}-x_{2}\right) v, x_{2}\right) \Phi\left(x_{1}\right) \quad \text { for all } v \in V .
$$

A pseudo-endomorphism $\Phi(x)$ is called a pseudo-automorphism if there exists a pseudo-endomorphism $\Psi(x)$ such that $\Phi(x) \Psi(x) v=v=\Psi(x) \Phi(x) v$ for $v \in V$. We say that pseudo-endomorphisms $\Phi(x)$ and $\Psi(x)$ commute if $\Phi\left(x_{1}\right) \Psi\left(x_{2}\right)=$ $\Psi\left(x_{2}\right) \Phi\left(x_{1}\right)$.

The following is straightforward (see [Li5]):

Proposition 3.14. Let $V$ be a nonlocal vertex algebra. A pseudo-endomorphism of $V$ exactly amounts to a nonlocal-vertex-algebra homomorphism from $V$ to the tensor product nonlocal vertex algebra $V \otimes \mathbb{C}((x))$, where $\mathbb{C}((x))$ is viewed as a vertex algebra with 1 as the vacuum vector and

$$
Y(f(x), z) g(x)=f(x-z) g(x) \quad \text { for } f(x), g(x) \in \mathbb{C}((x)) .
$$

We have the following results for the quantum vertex algebra $V_{\mathbf{Q}}$ :

Lemma 3.15. Let $\mathbf{Q}=\left(q_{i j}\right)$ be an $l \times l$ matrix as before. For any $\left(p_{1}(x), \ldots, p_{l}(x)\right) \in$ $\mathbb{C}((x))^{l}$ with $p_{i}(x) \neq 0$ for all $i$, there exists a pseudo-automorphism $\Phi(x)$ of $V_{\mathbf{Q}}$ such that

$$
\Phi(x)\left(u^{(i)}\right)=u^{(i)} \otimes p_{i}(x), \quad \Phi(x)\left(v^{(i)}\right)=v^{(i)} \otimes p_{i}(x)^{-1} \quad \text { for } i=1, \ldots, l .
$$

Furthermore, all such pseudo-automorphisms commute. 
Proof. Denote by $\hat{Y}$ the vertex operator map for the tensor product nonlocal vertex algebra $V_{\mathbf{Q}} \otimes \mathbb{C}((x))$. From definition we have

$$
\hat{Y}(a \otimes f(x), z)=Y(a, z) \otimes f(x-z) \quad \text { for } a \in V_{\mathbf{Q}}, f(x) \in \mathbb{C}((x)) .
$$

The following relations hold:

$$
\begin{aligned}
& \hat{Y}\left(u^{(i)} \otimes p_{i}(x), x_{1}\right) \hat{Y}\left(u^{(j)} \otimes p_{j}(x), x_{2}\right) \\
& =Y\left(u^{(i)}, x_{1}\right) Y\left(u^{(j)}, x_{2}\right) \otimes p_{i}\left(x-x_{1}\right) p_{j}\left(x-x_{2}\right) \\
& =q_{i j} Y\left(u^{(j)}, x_{2}\right) Y\left(u^{(i)}, x_{1}\right) \otimes p_{j}\left(x-x_{2}\right) p_{i}\left(x-x_{1}\right) \\
& =q_{i j} \hat{Y}\left(u^{(j)} \otimes p_{j}(x), x_{2}\right) \hat{Y}\left(u^{(i)} \otimes p_{i}(x), x_{1}\right) \text {, } \\
& \hat{Y}\left(v^{(i)} \otimes p_{i}(x)^{-1}, x_{1}\right) \hat{Y}\left(v^{(j)} \otimes p_{j}(x)^{-1}, x_{2}\right) \\
& =q_{i j} \hat{Y}\left(v^{(j)} \otimes p_{j}(x)^{-1}, x_{2}\right) \hat{Y}\left(v^{(i)} \otimes p_{i}(x)^{-1}, x_{1}\right) \text {, } \\
& \hat{Y}\left(u^{(i)} \otimes p_{i}(x), x_{1}\right) \hat{Y}\left(v^{(j)} \otimes p_{j}(x)^{-1}, x_{2}\right) \\
& -q_{j i} \hat{Y}\left(v^{(j)} \otimes p_{j}(x)^{-1}, x_{2}\right) \hat{Y}\left(u^{(i)} \otimes p_{i}(x), x_{1}\right) \\
& =\left(Y\left(u^{(i)}, x_{1}\right) Y\left(v^{(j)}, x_{2}\right)-q_{j i} Y\left(v^{(j)}, x_{2}\right) Y\left(v^{(i)}, x_{1}\right)\right) \otimes p_{i}\left(x-x_{1}\right) p_{j}^{-1}\left(x-x_{2}\right) \\
& =\delta_{i j} p_{i}\left(x-x_{1}\right) p_{j}^{-1}\left(x-x_{2}\right) x_{2}^{-1} \delta\left(\frac{x_{1}}{x_{2}}\right) \\
& =\delta_{i j} p_{i}\left(x-x_{2}\right) p_{j}^{-1}\left(x-x_{2}\right) x_{2}^{-1} \delta\left(\frac{x_{1}}{x_{2}}\right) \\
& =\delta_{i j} x_{2}^{-1} \delta\left(\frac{x_{1}}{x_{2}}\right) \text {. }
\end{aligned}
$$

In view of Proposition 3.8, there exists a nonlocal-vertex-algebra homomorphism $\psi$ from $V_{\mathbf{Q}}$ into $V_{\mathbf{Q}} \otimes \mathbb{C}((x))$ such that

$$
\psi\left(u^{(i)}\right)=u^{(i)} \otimes p_{i}(x), \quad \psi\left(v^{(i)}\right)=v^{(i)} \otimes p_{i}(x)^{-1} \quad \text { for } i=1, \ldots, l .
$$

The map $\psi: V_{\mathbf{Q}} \rightarrow V_{\mathbf{Q}} \otimes \mathbb{C}((x))$, alternatively denoted by $\Phi(x)$, is a pseudoautomorphism of $V$ satisfying the required property (recall Proposition 3.14). The rest is clear.

\section{Zamolodchikov-Faddeev type quantum vertex algebras}

The associative algebras $\mathcal{A}_{\mathrm{Q}}$, which were studied in the previous section, are actually the simplest Zamolodchikov-Faddeev algebras. A general Zamolodchikov-Faddeev algebra is associated with a quantum Yang-Baxter operator $\mathcal{S}\left(x_{1}, x_{2}\right)$ (with two 
spectral parameters) on a finite-dimensional vector space. In [Li8], for any finitedimensional vector space $H$ equipped with a bilinear form and a linear map $\mathcal{S}(x)$ : $H \otimes H \otimes \mathbb{C}[[x]]$, we constructed a "universal" weak quantum vertex algebra $V(H, \mathcal{S})$ with certain generators and defining relations. Then we studied a special family of $V(H, \mathcal{S})$ and proved that $V(H, \mathcal{S})$ are nondegenerate quantum vertex algebras. In this section, we study the case in which $\mathcal{S}(x)$ is diagonalizable. By using the results of Sections 2 and 3 we prove that either $V(H, S)$ is zero, or an irreducible quantum vertex algebra with a normal basis in a certain sense. Furthermore, we prove that $V(H, S)$ are indeed nonzero for a certain family.

First we recall from [Li8] the following notion:

Definition 4.1. Let $H$ be a vector space equipped with a bilinear form $\langle\cdot, \cdot\rangle$ and let $\mathcal{S}(x): H \otimes H \rightarrow H \otimes H \otimes \mathbb{C}((x))$ be a linear map. An $(H, \mathcal{S})$-module is a module $W$ for the (free) tensor algebra $T\left(H \otimes \mathbb{C}\left[t, t^{-1}\right]\right)$ such that for any $a \in H, w \in W$,

$$
a(m) w=0 \quad \text { for } m \text { sufficiently large, }
$$

where $a(m)$ denotes the operator on $W$ corresponding to $a \otimes t^{m}$, and such that

$$
a\left(x_{1}\right) b\left(x_{2}\right) w-\sum_{i=1}^{r} f_{i}\left(x_{2}-x_{1}\right) b^{(i)}\left(x_{2}\right) a^{(i)}\left(x_{1}\right) w=x_{2}^{-1} \delta\left(\frac{x_{1}}{x_{2}}\right)\langle a, b\rangle w
$$

for $a, b \in H, w \in W$, where $u(x)=\sum_{m \in \mathbb{Z}} u(m) x^{-m-1}$ for $u \in H$ and

$$
\mathcal{S}(x)(b \otimes a)=\sum_{i=1}^{r} b^{(i)} \otimes a^{(i)} \otimes f_{i}(x) .
$$

(Notice that the condition (4.1) guarantees that for any $m, n \in \mathbb{Z}$, the coefficient of $x_{1}^{m} x_{2}^{n}$ in the left side of (4.2) is a finite sum in $W$.)

Let $W$ be an $(H, \mathcal{S})$-module. A vector $e \in W$ is called a vacuum vector if

$$
a(m) e=0 \quad \text { for all } a \in H, m \geq 0 .
$$

A vacuum $(H, \mathcal{S})$-module is an $(H, \mathcal{S})$-module $W$ equipped with a vacuum vector $e$ that generates $W$. We also denote the vacuum module by $(W, e)$. The notion of a universal vacuum $(H, \mathcal{S})$-module is defined in the obvious way. If $\mathcal{S}(x): H \otimes H \rightarrow$ $H \otimes H \otimes \mathbb{C}[[x]]$, universal vacuum $(H, \mathcal{S})$-modules exist, as was shown in [Li8] by the following tautological construction: Let $T\left(H \otimes \mathbb{C}\left[t, t^{-1}\right]\right)^{+}$be the subspace of $T\left(H \otimes \mathbb{C}\left[t, t^{-1}\right]\right)$, spanned by the vectors

$$
\left(a^{(1)} \otimes t^{n_{1}}\right) \cdots\left(a^{(r)} \otimes t^{n_{r}}\right)
$$

for $r \geq 1, a^{(i)} \in H, n_{i} \in \mathbb{Z}$ with $n_{1}+\cdots+n_{r} \geq 0$. Set

$$
J=T\left(H \otimes \mathbb{C}\left[t, t^{-1}\right]\right) T\left(H \otimes \mathbb{C}\left[t, t^{-1}\right]\right)^{+},
$$


a left ideal of $T\left(H \otimes \mathbb{C}\left[t, t^{-1}\right]\right)$, and then set

$$
\tilde{V}(H, \mathcal{S})=T\left(H \otimes \mathbb{C}\left[t, t^{-1}\right]\right) / J,
$$

a left $T\left(H \otimes \mathbb{C}\left[t, t^{-1}\right]\right)$-module. Clearly, the condition (4.1) holds. Define $V(H, \mathcal{S})$ to be the quotient module of $\tilde{V}(H, \mathcal{S})$ modulo the relations (4.2). Let 1 denote the image in $V(H, \mathcal{S})$ of 1.

The following result was established in [Li8] (Propositions 2.18 and 4.3):

Proposition 4.2. Let $H$ be a vector space equipped with a bilinear form $\langle\cdot, \cdot\rangle$ and let $\mathcal{S}(x): H \otimes H \rightarrow H \otimes H \otimes \mathbb{C}[[x]]$ be a linear map. Then $(V(H, \mathcal{S}), \mathbf{1})$ is a universal vacuum $(H, \mathcal{S})$-module and there exists a unique weak quantum vertex algebra structure on $V(H, \mathcal{S})$ with $\mathbf{1}$ as the vacuum vector such that

$$
Y(a(-1) \mathbf{1}, x)=a(x) \quad \text { for } a \in H .
$$

Furthermore, on any $(H, \mathcal{S})$-module $W$ there exists a unique $V(H, \mathcal{S})$-module structure $Y_{W}$ such that

$$
Y_{W}(a(-1) \mathbf{1}, x)=a(x) \quad \text { for } a \in H .
$$

Remark 4.3. Note that it was assumed in ([Li8], Proposition 2.18) that the linear map from $H$ to $V(H, \mathcal{S})$, sending $a$ to $a(-1) \mathbf{1}$ for $a \in H$, is injective. In fact, this assumption was not used in the proof. If the linear map $a \in H \mapsto a(-1) \mathbf{1} \in V(H, \mathcal{S})$ is injective, $H$ can be identified with a subspace of $V(H, \mathcal{S})$ and $H$ is a generating subspace. In general, the subspace $\{a(-1) \mathbf{1} \mid a \in H\}$ is a generating subspace.

Remark 4.4. The nonlocal vertex algebra $V(H, \mathcal{S})$ is universal in the sense that for any given nonlocal vertex algebra $V$ and for any given linear map $\phi: H \rightarrow V$ satisfying

$$
\begin{aligned}
& Y\left(\phi(u), x_{1}\right) Y\left(\phi(v), x_{2}\right)-\sum_{i=1}^{r} f_{i}\left(x_{2}-x_{1}\right) Y\left(\phi\left(v^{(i)}\right), x_{2}\right) Y\left(\phi\left(u^{(i)}\right), x_{1}\right) \\
& \quad=\langle u, v\rangle x_{2}^{-1} \delta\left(\frac{x_{1}}{x_{2}}\right)
\end{aligned}
$$

for $u, v \in H$, where

$$
\mathcal{S}(x)(v \otimes u)=\sum_{i=1}^{r} v^{(i)} \otimes u^{(i)} \otimes f_{i}(x) \in H \otimes H \otimes \mathbb{C}[[x]],
$$

there exists a unique nonlocal-vertex-algebra homomorphism from $V(H, \mathcal{S})$ to $V$, sending $a(-1) 1$ to $\phi(a)$ for $a \in H$. Indeed, the relation (4.4) implies that $V$ is an $(H, \mathcal{S})$-module with $a(x)=Y(\phi(a), x)$ for $a \in H$. Clearly, $\mathbf{1}$ is a vacuum vector of $V$ viewed as an $(H, \mathcal{S})$-module. It follows that the $(H, \mathcal{S})$-submodule of $V$ generated 
from 1 is a vacuum module. Then there exists an $(H, \mathcal{S})$-module homomorphism $\psi$ from $V(H, \mathcal{S})$ to $V$, sending the vacuum vector to the vacuum vector. Since $\{a(-1) \mathbf{1} \mid a \in H\}$ generates $V(H, \mathcal{S})$ as a nonlocal vertex algebra, it follows that $\psi$ is a nonlocal-vertex-algebra homomorphism, where

$$
\psi(a(-1) \mathbf{1})=\operatorname{Res}_{x} x^{-1} \psi(Y(a(-1) \mathbf{1}, x) \mathbf{1})=\operatorname{Res}_{x} x^{-1} Y(\phi(a), x) \mathbf{1}=\phi(a)
$$

for $a \in H$. The uniqueness is clear.

The following is our first result of this section:

Theorem 4.5. Let $H$ be a finite-dimensional vector space equipped with a bilinear form $\langle\cdot, \cdot\rangle$ and let $\mathcal{S}(x): H \otimes H \rightarrow H \otimes H \otimes \mathbb{C}[[x]]$ be a linear map. Suppose that there exists a basis $\left\{u^{(1)}, \ldots, u^{(l)}, v^{(1)}, \ldots, v^{(l)}\right\}$ of $H$ such that

$$
\left\langle u^{(i)}, u^{(j)}\right\rangle=0=\left\langle v^{(i)}, v^{(j)}\right\rangle, \quad\left\langle u^{(i)}, v^{(j)}\right\rangle=\delta_{i j}=-q_{i i}\left\langle v^{(i)}, u^{(j)}\right\rangle
$$

and

$$
\begin{aligned}
& \mathcal{S}(0)\left(u^{(i)} \otimes u^{(j)}\right)=q_{j i}\left(u^{(i)} \otimes u^{(j)}\right), \quad \mathcal{S}(0)\left(v^{(i)} \otimes v^{(j)}\right)=q_{j i}\left(v^{(i)} \otimes v^{(j)}\right), \\
& \mathcal{S}(0)\left(u^{(i)} \otimes v^{(j)}\right)=q_{i j}\left(u^{(i)} \otimes v^{(j)}\right), \quad \mathcal{S}(0)\left(v^{(j)} \otimes u^{(i)}\right)=q_{i j}\left(v^{(j)} \otimes u^{(i)}\right)
\end{aligned}
$$

where $q_{i j} \in \mathbb{C}$ with $q_{i j} q_{j i}=1$ for $i, j=1, \ldots, l$. Assume that $V(H, \mathcal{S})$ is not zero. Then $V(H, \mathcal{S})$ is an irreducible quantum vertex algebra with $\operatorname{Gr}_{F}(V(H, \mathcal{S}))=V_{\mathbf{Q}}$ where $\mathbf{Q}=\left(q_{i j}\right)_{i, j=1}^{l}$ and $F$ is the increasing filtration of $V$, defined in Lemma 2.9, associated with the generating subspace $T=\{a(-1) \mathbf{1} \mid a \in H\}$. Furthermore, $\mathcal{S}(x)$ must be a unitary rational quantum Yang-Baxter operator.

Proof. We first prove $\operatorname{Gr}_{F}(V(H, \mathcal{S}))=V_{\mathbf{Q}}$. Recall that $V(H, \mathcal{S})$ is generated by $a(-1) \mathbf{1}$ for $a \in H$, where $Y(a(-1) \mathbf{1}, x)=a(x)$. By definition, for $n<0, F_{n}$ is linearly spanned by the vectors

$$
a^{(1)}\left(m_{1}\right) \cdots a^{(r)}\left(m_{r}\right) \mathbf{1}
$$

for $r \geq 1, a^{(i)} \in H, m_{i} \in \mathbb{Z}$ with $m_{1}+\cdots+m_{r} \geq-n$, and for $n \geq 0, F_{n}$ is linearly spanned by 1 and the vectors of the same form. By Corollary 4.2 of [Li8], we have

$$
h^{(1)}\left(m_{1}\right) \cdots h^{(r)}\left(m_{r}\right) \mathbf{1}=0
$$

for $r \geq 1, h^{(1)}, \ldots, h^{(r)} \in H, m_{1}, \ldots, m_{r} \in \mathbb{Z}$ with $m_{1}+\cdots+m_{r} \geq 0$. Consequently, $F_{n}=0$ for $n \leq-1$ and $F_{0}=\mathbb{C} \mathbf{1}(\neq 0)$. Then $\operatorname{Gr}_{F}(V(H, \mathcal{S})) \neq 0$.

Notice that $a(-1) \mathbf{1} \in F_{1}$ for $a \in H$. For $1 \leq i \leq l$, set

$$
\bar{u}^{(i)}=u^{(i)}(-1) \mathbf{1}+F_{0}, \quad \bar{v}^{(i)}=v^{(i)}(-1) \mathbf{1}+F_{0} \in F_{1} / F_{0} \subset \operatorname{Gr}_{F}(V(H, \mathcal{S})) .
$$


Then $\operatorname{Gr}_{F}(V(H, \mathcal{S}))$ is generated by $\bar{u}^{(i)}, \bar{v}^{(i)}$ for $1 \leq i \leq l$ and we have

$$
\begin{aligned}
& Y\left(\bar{u}^{(i)}, x_{1}\right) Y\left(\bar{u}^{(j)}, x_{2}\right)=q_{i j} Y\left(\bar{u}^{(j)}, x_{2}\right) Y\left(\bar{u}^{(i)}, x_{1}\right), \\
& Y\left(\bar{v}^{(i)}, x_{1}\right) Y\left(\bar{v}^{(j)}, x_{2}\right)=q_{i j} Y\left(\bar{v}^{(j)}, x_{2}\right) Y\left(\bar{v}^{(i)}, x_{1}\right), \\
& Y\left(\bar{u}^{(i)}, x_{1}\right) Y\left(\bar{v}^{(j)}, x_{2}\right)-q_{j i} Y\left(\bar{v}^{(j)}, x_{2}\right) Y\left(\bar{u}^{(i)}, x_{1}\right)=\delta_{i j} x_{1}^{-1} \delta\left(\frac{x_{2}}{x_{1}}\right)
\end{aligned}
$$

for $i, j=1, \ldots, l$. In view of Proposition 3.8 , there exists a nonlocal-vertex-algebra homomorphism $\psi$ from $V_{\mathbf{Q}}$ onto $\operatorname{Gr}_{F}(V(H, \mathcal{S}))$ such that

$$
\psi\left(a^{(i)}\right)=\bar{u}^{(i)}, \quad \psi\left(b^{(i)}\right)=\bar{v}^{(i)} \quad \text { for } i=1, \ldots, l .
$$

Since $V_{\mathbf{Q}}$ is simple and $\operatorname{Gr}_{F}(V(H, \mathcal{S})) \neq 0, \psi$ must be an isomorphism. This proves $\operatorname{Gr}_{F}(V(H, \mathcal{S}))=V_{\mathbf{Q}}$. As $V_{\mathbf{Q}}$ is irreducible, by Proposition 2.11, $V(H, \mathcal{S})$ is irreducible. Then $V(H, \mathcal{S})$ is an irreducible quantum vertex algebra. As $V(H, \mathcal{S})$ is a nondegenerate quantum vertex algebra, it follows that $\mathcal{S}$ must be a unitary rational quantum Yang-Baxter operator on $H$.

Remark 4.6. Here we correct an error in [Li8]. In Theorem 4.4 of [Li8], it was assumed that $H$ is a finite-dimensional vector space equipped with a bilinear form $\langle\cdot, \cdot\rangle$ and $\mathcal{S}(x): H \otimes H \rightarrow H \otimes H \otimes \mathbb{C}[[x]]$ is a linear map with $\mathcal{S}(0)=1$. Furthermore it was assumed that $\operatorname{Gr}_{F}(V(H, \mathcal{S}))$ is linearly isomorphic to the symmetric algebra over the space $H \otimes t^{-1} \mathbb{C}\left[t^{-1}\right]$ under a canonical map. It was then claimed that $V(H, \mathcal{S})$ is a nondegenerate quantum vertex algebra. The reasoning therein used Proposition 2.18 of [Li8], which claims that for a nonlocal vertex algebra $V$ with a generating subset $T$, if $\operatorname{Gr}_{E}(V)$ is nondegenerate, then $V$ is nondegenerate, where $E$ is the increasing filtration of $V$, constructed in Lemma 2.6. The discrepancy is that the filtration $F$ in the assumption is different from $E$ in Proposition 2.18. Now, we modify this theorem by adding the assumption that $\langle\cdot, \cdot\rangle$ is a nondegenerate skewsymmetric bilinear form. This modified theorem becomes a special case of Theorem 4.5 which uses Proposition 2.11 of the current paper, instead of Proposition 2.18 of [Li8]. For the example that was constructed in [Li8], $H=U \oplus U^{*}$ for some vector space $U$, equipped with the standard nondegenerate skew-symmetric bilinear form, so the modified theorem is applicable.

For the rest of this section we consider the special case with $\mathcal{S}(x)$ diagonal.

Definition 4.7. Let $l$ be a positive integer and let $\mathbf{Q}(x)=\left(q_{i j}(x)\right)$ be an $l \times l$ matrix over $\mathbb{C}[[x]]$ such that

$$
q_{i j}(x) q_{j i}(-x)=1 \quad \text { for } 1 \leq i, j \leq l .
$$

Let $H$ be a $2 l$-dimensional vector space with a basis $\left\{a^{(1)}, \ldots, a^{(l)}, b^{(1)}, \ldots, b^{(l)}\right\}$. Define a linear map $\mathcal{S}(x): H \otimes H \rightarrow H \otimes H \otimes \mathbb{C}[[x]]$ by

$$
\begin{aligned}
& \mathcal{S}(x)\left(a^{(i)} \otimes a^{(j)}\right)=q_{j i}(x)\left(a^{(i)} \otimes a^{(j)}\right), \quad \mathcal{S}(x)\left(b^{(i)} \otimes b^{(j)}\right)=q_{j i}(x)\left(b^{(i)} \otimes b^{(j)}\right), \\
& \mathcal{S}(x)\left(a^{(i)} \otimes b^{(j)}\right)=q_{i j}(-x)\left(a^{(i)} \otimes b^{(j)}\right), \quad \mathcal{S}(x)\left(b^{(i)} \otimes a^{(j)}\right)=q_{i j}(-x)\left(b^{(i)} \otimes a^{(j)}\right)
\end{aligned}
$$


for $i, j=1, \ldots, l$, and equip $H$ with a bilinear form $\langle\cdot, \cdot\rangle$ defined by

$$
\left\langle a^{(i)}, a^{(j)}\right\rangle=\left\langle b^{(i)}, b^{(j)}\right\rangle=0 \quad \text { and } \quad\left\langle a^{(i)}, b^{(j)}\right\rangle=\delta_{i j}=-q_{i i}(0)\left\langle b^{(j)}, a^{(i)}\right\rangle .
$$

We define $V_{\mathbf{Q}(x)}$ to be the weak quantum vertex algebra $V(H, \mathcal{S})$ associated with this pair $(H, \mathcal{S})$ from $\mathbf{Q}(x)$. That is, $V_{\mathbf{Q}(x)}$ is a universal weak quantum vertex algebra with generators $a^{(i)}, b^{(i)}(i=1, \ldots, l)$, satisfying relations

$$
\begin{aligned}
& Y\left(a^{(i)}, x_{1}\right) Y\left(a^{(j)}\left(x_{2}\right)=q_{i j}\left(x_{2}-x_{1}\right) Y\left(a^{(j)}, x_{2}\right) Y\left(a^{(i)}, x_{1}\right),\right. \\
& Y\left(b^{(i)}, x_{1}\right) Y\left(b^{(j)}, x_{2}\right)=q_{i j}\left(x_{2}-x_{1}\right) Y\left(b^{(j)}, x_{2}\right) Y\left(b^{(i)}, x_{1}\right), \\
& Y\left(a^{(i)}, x_{1}\right) Y\left(b^{(j)}, x_{2}\right)-q_{j i}\left(x_{1}-x_{2}\right) Y\left(b^{(j)}, x_{2}\right) Y\left(a^{(i)}, x_{1}\right)=\delta_{i j} x_{1}^{-1} \delta\left(\frac{x_{2}}{x_{1}}\right)
\end{aligned}
$$

for $i, j=1, \ldots, l$.

With this notion, as an immediate consequence of Theorem 4.5 we have:

Corollary 4.8. Let $l$ be a positive integer and let $\mathbf{Q}(x)=\left(q_{i j}(x)\right)$ be an $l \times l$ matrix in $\mathbb{C}[[x]]$ satisfying (4.5). Suppose that $V$ is a nonzero weak quantum vertex algebra with a generating subset $T=\left\{u^{(i)}, v^{(i)} \mid i=1, \ldots, l\right\}$, satisfying the relations

$$
\begin{aligned}
& Y\left(u^{(i)}, x_{1}\right) Y\left(u^{(j)}\left(x_{2}\right)=q_{i j}\left(x_{2}-x_{1}\right) Y\left(u^{(j)}, x_{2}\right) Y\left(u^{(i)}, x_{1}\right),\right. \\
& Y\left(v^{(i)}, x_{1}\right) Y\left(v^{(j)}, x_{2}\right)=q_{i j}\left(x_{2}-x_{1}\right) Y\left(v^{(j)}, x_{2}\right) Y\left(v^{(i)}, x_{1}\right), \\
& Y\left(u^{(i)}, x_{1}\right) Y\left(v^{(j)}, x_{2}\right)-q_{j i}\left(x_{1}-x_{2}\right) Y\left(v^{(j)}, x_{2}\right) Y\left(u^{(i)}, x_{1}\right)=\delta_{i j} x_{1}^{-1} \delta\left(\frac{x_{2}}{x_{1}}\right)
\end{aligned}
$$

for $i, j=1, \ldots, l$. Then $V$ is an irreducible quantum vertex algebra with $G_{F}(V)=$ $V_{\mathbf{Q}(0)}$, where $F$ is the increasing filtration of $V$, defined in Lemma 2.9, associated with the generating set $T$. Furthermore, such a nonzero quantum vertex algebra $V$, if it exists, is unique up to isomorphism.

The following is our second main result of this section:

Theorem 4.9. Let $\mathbf{Q}(x)=\left(q_{i j}(x)\right)_{i, j=1}^{l}$ with

$$
q_{i j}(x)=q_{i j} p_{i j}(-x) / p_{i j}(x),
$$

where $q_{i j} \in \mathbb{C}, p_{i j}(x) \in \mathbb{C}[[x]]$ satisfy that $q_{i j} q_{j i}=1, p_{i j}(0)=1$ and $p_{i j}(x)=p_{j i}(x)$ for all $i, j=1, \ldots, l$. Then $V_{\mathbf{Q}(x)}$ is a (nonzero) irreducible quantum vertex algebra with $\operatorname{Gr}_{F}\left(V_{\mathbf{Q}(x)}\right)=V_{\mathbf{Q}(0)}$.

Proof. By Lemma 3.15, there exist mutually commuting pseudo-automorphisms $\Phi_{i}(x)$ for $1 \leq i \leq l$ of $V_{\mathbf{Q}(0)}$ such that

$$
\Phi_{i}(x)\left(u^{(j)}\right)=u^{(j)} \otimes p_{i j}(x), \quad \Phi_{i}(x)\left(v^{(j)}\right)=v^{(j)} \otimes p_{i j}(x)^{-1} \quad \text { for } j=1, \ldots, l .
$$


Set

$$
a^{(i)}(x)=Y\left(u^{(i)}, x\right) \Phi_{i}(x), \quad b^{(i)}(x)=Y\left(v^{(i)}, x\right) \Phi_{i}^{-1}(x)
$$

for $i=1, \ldots, l$. We have

$$
\begin{aligned}
a^{(i)}\left(x_{1}\right) a^{(j)}\left(x_{2}\right) & =Y\left(u^{(i)}, x_{1}\right) \Phi_{i}\left(x_{1}\right) Y\left(u^{(j)}, x_{2}\right) \Phi_{j}\left(x_{2}\right) \\
& =p_{i j}\left(x_{1}-x_{2}\right) Y\left(u^{(i)}, x_{1}\right) Y\left(u^{(j)}, x_{2}\right) \Phi_{i}\left(x_{1}\right) \Phi_{j}\left(x_{2}\right) \\
& =q_{i j} p_{i j}\left(x_{1}-x_{2}\right) Y\left(u^{(j)}, x_{2}\right) Y\left(u^{(i)}, x_{1}\right) \Phi_{j}\left(x_{2}\right) \Phi_{i}\left(x_{1}\right) \\
& =q_{i j} p_{i j}\left(x_{1}-x_{2}\right) p_{j i}\left(x_{2}-x_{1}\right)^{-1} a^{(j)}\left(x_{2}\right) a^{(i)}\left(x_{1}\right) \\
& =q_{i j}\left(x_{2}-x_{1}\right) a^{(j)}\left(x_{2}\right) a^{(i)}\left(x_{1}\right), \\
b^{(i)}\left(x_{1}\right) b^{(j)}\left(x_{2}\right) & =Y\left(v^{(i)}, x_{1}\right) \Phi_{i}^{-1}\left(x_{1}\right) Y\left(v^{(j)}, x_{2}\right) \Phi_{j}^{-1}\left(x_{2}\right) \\
= & p_{i j}\left(x_{1}-x_{2}\right) Y\left(v^{(i)}, x_{1}\right) Y\left(v^{(j)}, x_{2}\right) \Phi_{i}^{-1}\left(x_{1}\right) \Phi_{j}^{-1}\left(x_{2}\right) \\
= & q_{i j} p_{i j}\left(x_{1}-x_{2}\right) Y\left(v^{(j)}, x_{2}\right) Y\left(v^{(i)}, x_{1}\right) \Phi_{j}^{-1}\left(x_{2}\right) \Phi_{i}^{-1}\left(x_{1}\right) \\
= & q_{i j} p_{i j}\left(x_{1}-x_{2}\right) p_{j i}\left(x_{2}-x_{1}\right)^{-1} b^{(j)}\left(x_{2}\right) b^{(i)}\left(x_{1}\right) \\
= & q_{i j}\left(x_{2}-x_{1}\right) b^{(j)}\left(x_{2}\right) b^{(i)}\left(x_{1}\right), \\
= & a^{(i)}\left(x_{1}\right) b^{(j)}\left(x_{2}\right)-q_{j i} \frac{p_{j i}\left(x_{2}-x_{1}\right)}{p_{i j}\left(x_{1}-x_{2}\right)} b^{(j)}\left(x_{2}\right) a^{(i)}\left(x_{1}\right) \\
= & p_{i j}\left(x_{1}-x_{2}\right)^{-1} Y\left(u^{(i)}, x_{1}\right) Y\left(v^{(j)}, x_{2}\right) \Phi_{i}\left(x_{1}\right) \Phi_{j}^{-1}\left(x_{2}\right) \\
& -q_{j i} p_{i j}\left(x_{1}-x_{2}\right)^{-1} Y\left(v^{(j)}, x_{2}\right) Y\left(u^{(i)}, x_{1}\right) \Phi_{j}^{-1}\left(x_{2}\right) \Phi_{i}\left(x_{1}\right) \\
= & \delta_{i j} x_{2}^{-1} \delta\left(\frac{x_{1}}{x_{2}}\right) p_{i j}\left(x_{1}-x_{2}\right)^{-1} \Phi_{j}^{-1}\left(x_{2}\right) \Phi_{i}\left(x_{1}\right) \\
= & \delta_{i j} x_{2}^{-1} \delta\left(\frac{x_{1}}{x_{2}}\right) p_{i j}(0)^{-1} \Phi_{j}^{-1}\left(x_{2}\right) \Phi_{i}\left(x_{2}\right) \\
\delta_{i j} x_{2}^{-1} \delta & \left(\frac{x_{1}}{x_{2}}\right) \cdot
\end{aligned}
$$

That is, we have defined an $(H, \mathcal{S})$-module structure on $V_{\mathbf{Q}(0)}$. Using the fact that $\Phi_{i}(x)(\mathbf{1})=\mathbf{1}$ for $i=1, \ldots, l$, we see that $\mathbf{1}$ is a vacuum vector of the $(H, \mathcal{S})$-module $V_{\mathbf{Q}(0)}$. We claim that the $(H, \mathcal{S})$-module $V_{\mathbf{Q}(0)}$ is generated by $\mathbf{1}$, so that $V_{\mathbf{Q}(0)}$ is a nonzero vacuum $(H, \mathcal{S})$-module, which implies that $V_{\mathbf{Q}(x)} \neq 0$. Let $E$ be the submodule of the $(H, \mathcal{S})$-module $V_{\mathbf{Q}(0)}$ generated by $\mathbf{1}$. We have $\Phi_{i}(x) \mathbf{1}=\mathbf{1}$,

$$
\Phi_{i}(x) a^{(j)}\left(x_{1}\right)=p_{i j}\left(x-x_{1}\right) a^{(j)}\left(x_{1}\right) \Phi_{i}(x), \quad \Phi_{i}(x) b^{(j)}\left(x_{1}\right)=p_{i j}\left(x-x_{1}\right)^{-1} b^{(j)}\left(x_{1}\right) \Phi_{i}(x)
$$

for $i, j=1, \ldots, l$. It follows from induction that $\Phi_{i}(x) E \subset E((x))$ for $1 \leq i \leq l$. Similarly, we have $\Phi_{i}(x)^{-1} E \subset E((x))$. As

$$
Y\left(u^{(i)}, x\right)=a^{(i)}(x) \Phi_{i}(x)^{-1}, \quad Y\left(v^{(i)}, x\right)=b^{(i)}(x) \Phi_{i}(x),
$$


it follows that $E$ is closed under the components of $Y\left(u^{(i)}, x\right)$ and $Y\left(v^{(i)}, x\right)$ for $i=1, \ldots, l$. Consequently, $E=V_{\mathbf{Q}(0)}$, as claimed. This completes the proof.

Example 4.10. Let $f(x) \in \mathbb{C}[[x]]$ with $f(0)=1$. In view of Theorem 4.9, there exists a (nonzero) irreducible quantum vertex algebra $V$ with a generating set $\{a, b\}$ such that

$$
\begin{aligned}
& Y\left(a, x_{1}\right) Y\left(a, x_{2}\right)= \pm f\left(x_{1}-x_{2}\right) f\left(x_{2}-x_{1}\right)^{-1} Y\left(a, x_{2}\right) Y\left(a, x_{1}\right) \\
& Y\left(b, x_{1}\right) Y\left(b, x_{2}\right)= \pm f\left(x_{1}-x_{2}\right) f\left(x_{2}-x_{1}\right)^{-1} Y\left(b, x_{2}\right) Y\left(b, x_{1}\right) \\
& Y\left(a, x_{1}\right) Y\left(b, x_{2}\right) \mp f\left(x_{2}-x_{1}\right) f\left(x_{1}-x_{2}\right)^{-1} Y\left(b, x_{2}\right) Y\left(a, x_{1}\right)=x_{2}^{-1} \delta\left(\frac{x_{1}}{x_{2}}\right) .
\end{aligned}
$$

Furthermore, any nonzero nonlocal vertex algebra $V$ with a generating set $\{a, b\}$ satisfying the above relations is an irreducible quantum vertex algebra and such a nonzero quantum vertex algebra is unique up to isomorphism.

We furthermore consider certain nondegenerate, but not necessarily irreducible, quantum vertex algebras.

Proposition 4.11. Let $q_{i j} \in \mathbb{C}, p_{i j}(x) \in \mathbb{C}[[x]]$ be such that $q_{i j} q_{j i}=1, q_{i j}(0)=1$ and $q_{i j}(x)=q_{j i}(x)$ for $1 \leq i, j \leq l$. Suppose that $V$ is a weak quantum vertex algebra with a generating subset $\left\{u^{(1)}, \ldots, u^{(l)}\right\}$ such that

$$
Y\left(u^{(i)}, x_{1}\right) Y\left(u^{(j)}, x_{2}\right)=q_{i j} p_{i j}\left(x_{1}-x_{2}\right) p_{i j}\left(x_{2}-x_{1}\right)^{-1} Y\left(u^{(j)}, x_{2}\right) Y\left(u^{(i)}, x_{1}\right)
$$

for $1 \leq i, j \leq l$. Assume that $V$ has a normal basis in the sense that the normal vectors $X_{1} \cdots X_{l} \mathbf{1}$, where $X_{i}=u_{-n_{1}}^{(i)} \cdots u_{-n_{r}}^{(i)}$ for $r \geq 0, n_{i} \geq 1$ with $n_{1} \geq \cdots \geq n_{r}$ if $q_{i i}=1$ and $n_{1}>\cdots>n_{r}$ if $q_{i i}=-1$, form a basis of $V$. Then $V$ is a nondegenerate quantum vertex algebra. Furthermore, such a quantum vertex algebra exists and is unique up to isomorphism.

Proof. Set $\mathbf{Q}(x)=\left(q_{i j}(x)\right)_{i, j=1}^{l}$, where $q_{i j}(x)=q_{i j} p_{i j}(-x) p_{i j}(x)^{-1}$ for $i, j=1, \ldots, l$. By Theorem 4.9 and Corollary 4.8, we have a nondegenerate quantum vertex algebra $V_{\mathbf{Q}(x)}$ with $\operatorname{Gr}_{F}\left(V_{\mathbf{Q}(x)}\right)=V_{\mathbf{Q}(0)}$. Let $K$ be the subalgebgra of $V_{\mathbf{Q}(x)}$ generated by $u^{(1)}, \ldots, u^{(l)}$. Then $K$ is nondegenerate and has a normal basis.

Now, we prove that $V$ is isomorphic to $K$. Using the pair $(H, \mathcal{S})$ where $H=$ $\coprod_{i=1}^{l} \mathbb{C} u^{(i)}$ equipped with the zero form and $\mathcal{S}(x)\left(u^{(i)} \otimes u^{(j)}\right)=q_{i j}(x)\left(u^{(i)} \otimes u^{(j)}\right)$ for $1 \leq i, j \leq l$, we have a universal weak quantum vertex algebra $E(=V(H, \mathcal{S}))$ with generators $u^{(1)}, \ldots, u^{(l)}$ and with defining relation (4.9). Using the commutation relation (4.9) we see that $E$ is linearly spanned by all the normal vectors. As $K$ is a homomorphic image of $E$, it follows that $E$ also has a normal basis, so that $E$ is isomorphic to $K$. The same reasoning shows that $E$ is isomorphic to $V$. It follows that $E, K$ and $V$ are isomorphic and nondegenerate. 
Example 4.12. Let $V$ be a nonlocal vertex algebra with a generating set $\{e, f, h\}$, satisfying the following relations:

$$
\begin{aligned}
Y\left(e, x_{1}\right) Y\left(e, x_{2}\right) & =-\frac{1+x_{1}-x_{2}}{1-x_{1}+x_{2}} Y\left(e, x_{2}\right) Y\left(e, x_{1}\right), \\
Y\left(f, x_{1}\right) Y\left(f, x_{2}\right) & =-\frac{1-x_{1}+x_{2}}{1+x_{1}-x_{2}} Y\left(f, x_{2}\right) Y\left(f, x_{1}\right), \\
Y\left(h, x_{1}\right) Y\left(h, x_{2}\right) & =Y\left(h, x_{2}\right) Y\left(h, x_{1}\right) \\
Y\left(h, x_{1}\right) Y\left(e, x_{2}\right) & =-\frac{1+x_{1}-x_{2}}{1-x_{1}+x_{2}} Y\left(e, x_{2}\right) Y\left(h, x_{1}\right), \\
Y\left(h, x_{1}\right) Y\left(f, x_{2}\right) & =-\frac{1-x_{1}+x_{2}}{1+x_{1}-x_{2}} Y\left(f, x_{2}\right) Y\left(h, x_{1}\right), \\
Y\left(e, x_{1}\right) Y\left(f, x_{2}\right) & =Y\left(f, x_{2}\right) Y\left(e, x_{1}\right)
\end{aligned}
$$

Assume that $V$ has a basis consisting of the vectors

$$
e_{-m_{1}} \cdots e_{-m_{r}} h_{-n_{1}} \cdots h_{-n_{s}} f_{-k_{1}} \cdots f_{-k_{t}} \mathbf{1}
$$

for $r, s, t \geq 0, m_{1}>\cdots>m_{r} \geq 1, \quad n_{1}>\cdots>n_{s} \geq 1, \quad k_{1}>\cdots>k_{t} \geq 1$. Вy Proposition 4.11, $V$ is a nondegenerate quantum vertex algebra. This example is closely related to a quantum vertex algebra associated to double Yangian $D Y\left(s l_{2}\right)$ in [Li10].

\section{References}

[AB] I. Anguelova and M. Bergvelt, $H_{D^{-}}$Quantum vertex algebras and bicharacters, arXiv:0706.1528.

[BK] B. Bakalov and V. Kac, Field algebras, Internat. Math. Res. Notices 3 (2003) 123-159.

[D1] J. F. Duncan, Moonshine for Rudvalis's sporadic group I, arXiv: math/0609449.

[D2] J. F. Duncan, Moonshine for Rudvalis's sporadic group II, arXiv: math/0611355.

[EK] P. Etingof and D. Kazhdan, Quantization of Lie bialgebras, V, Selecta Math. (New Series) 6 (2000) 105-130.

[F] L. D. Faddeev, Quantum completely integrable models in field theory, Soviet Scientific Reviews Sect. C 1 (1980) 107-155.

[FFR] A. Feingold, I. B. Frenkel and J. F. Ries, Spinor construction of vertex operator algebras, triality, and $E_{8}^{(1)}$, Contemporary Math. 121, 1991. 
[Ka] V. Kac, Vertex Algebras for Beginners, University Lecture Series 10, Amer. Math. Soc., 1997.

[Ku] P. P. Kulish, Twist of quantum groups and noncommutative field theory, arXiv: hep-th/0606056.

[LL] J. Lepowsky and H.-S. Li, Introduction to Vertex Operator Algebras and Their Representations, Progress in Math. 227, Birkhäuser, Boston, 2004.

[Li1] H.-S. Li, Local systems of vertex operators, vertex superalgebras and modules, J. Pure Appl. Alg. 109 (1996) 143-195.

[Li2] H.-S. Li, Local systems of twisted vertex operators, vertex superalgebras and twisted modules, Contemporary Math. 193 (1996), 203-236.

[Li3] H.-S. Li, Axiomatic $G_{1}$-vertex algebras, Commun. Contemp. Math. 5 (2003) 281-327.

[Li4] H.-S. Li, Simple vertex operator algebras are nondegenerate, J. Alg. 267 (2003) 199-211.

[Li5] H.-S. Li, Pseudoderivations, pseudoautomorphisms and simple current modules for vertex operator algebras, Contemporary Math. 392 (2005) $55-65$.

[Li6] H.-S. Li, Nonlocal vertex algebras generated by formal vertex operators, Selecta Math. (New Series) 11 (2005) 349-397.

[Li7] H.-S. Li, A new construction of vertex algebras quasi modules for vertex algebras, Advances in Math. 202 (2006) 232-286.

[Li8] H.-S. Li, Constructing quantum vertex algebras, International Journal of Mathematics 17 (2006) 441-476.

[Li9] H.-S. Li, A smash product construction of nonlocal vertex algebras, Commun. Contemp. Math. 9 (2007) 605-637.

[Li10] H.-S. Li, Modules at infinity for quantum vertex algebras, submitted for publication; arXiv: 0705.0687.

[Ma1] Yu. I. Manin, Quantum Groups and Non-commutative Geometry, CRM Lecture Notes, 1989.

[Ma2] Yu. I. Manin, Topics in Noncommutative Geometry, Princeton University Press, 1991.

[M] S. Montgomery, Hopf Algebras and Their Actions on Rings, CBMS Regional Conference Series in Mathematics, Number 82, AMS, 1993. 
[S] M. E. Sweedler, Hopf Algebras, Benjamin, New York, 1969.

[MR] M. Mintchev and E. Ragoucy, Interplay between Zamolodchikov-Faddeev and reflection-transmission algebras, arXiv: math.QA/0306084.

[W] M. D. Weiner, Bosonic Construction of Vertex Operator Para-Algebras from Sympletic Affine Kac-Moody Algebras, Ph.D thesis, State University New York at Binghamton, 1994.

[ZZ] A. B. Zamolodchikov and A. B. Zamolodchikov, Factorized S-matrices in two-dimensions as the exact solutions of certain relativistic quantum field theory models, Ann. Phys. 120 (1979) 253-291. 